\title{
Carleman estimates for the regularization of ill-posed Cauchy problems
}

\author{
Michael V. Klibanov \\ Department of Mathematics \& Statistics, University of North Carolina at Charlot \\ Charlotte, NC, USA. Email: mklibanv@uncc.edu
}

\begin{abstract}
This is a survey, which is a continuation of the previous survey of the author about applications of Carleman estimates to Inverse Problems, J. Inverse and IllPosed Problems, 21, 477-560, 2013. It is shown here that Tikhonov functionals for some ill-posed Cauchy problems for linear PDEs can be generated by unbounded linear operators of those PDEs. These are those operators for which Carleman estimates are valid, e.g. elliptic, parabolic and hyperbolic operators of the second order. Convergence rates of minimizers are established using Carleman estimates. Generalizations to nonlinear inverse problems, such as problems of reconstructions of obstacles and coefficient inverse problems are discussed as well.
\end{abstract}

Keywords: Survey, Carleman estimates, Ill-Posed Cauchy problems, convergence rates.

AMS classification codes: $65 \mathrm{~N} 15,65 \mathrm{~N} 30,35 \mathrm{~J} 25$.

\section{Introduction}

This work is a survey, which is the continuation of the recent survey [56] of the author about applications of the method of Carleman estimates to inverse problems. Let $\Psi \subset \mathbb{R}^{n}$ be a bounded domain and $A$ be a linear Partial Differential Operator (PDO) of the second order acting in $\Psi$. Likewise, assume that this operator admits a Carleman estimate. In fact, the class of such operators is quite broad. Indeed, currently Carleman estimates are derived for three main classes of PDOs of the second order: elliptic, parabolic and hyperbolic ones, see, e.g. books of Beilina and Klibanov [9], Isakov [36], Klibanov and Timonov [52 and Lavrentiev, Romanov and Shishatskii 64 as well as the paper of Triggiani and Yao [72]. Therefore, results of this paper are quite general ones. Consider the Partial Differential Equation (PDE) $A u=f$ and an ill-posed Cauchy problem for it. Suppose that the Tikhonov functional for the solution of this problem is generated by the operator $A$. The current paper provides the positive answer for the following question: Can the solution of this Cauchy problem be approximated via the minimization of this functional?

Typically the regularization term is presented in the Tikhonov functional in a norm, which is stronger than the norm of the original space. Hence, we consider in our setting the domain of $A$ as $D(A)=H^{2}(\Psi) \subset L_{2}(\Psi)$ and $A: H^{2}(\Psi) \rightarrow L_{2}(\Psi)$. Thus, in this 
specific context $H^{2}(\Psi)$ is a linear set in $L_{2}(\Psi)$ and $\bar{H}^{2}(\Psi)=L_{2}(\Psi)$, where the closure is taken in the norm of $L_{2}(\Psi)$. Thus, we consider $A$ as an unbounded operator. As to the regularization theory for linear ill-posed problems with bounded linear operators, we refer to, e.g. the book of Ivanov, Vasin and Tanana [37].

For PDOs of the second order with above operators $A$, we present here a universal method of both constructions of Tikhonov functionals for solutions of ill-posed Cauchy problems for corresponding PDEs and estimating convergence rates of minimizers. First, we present our universal approach in which the operator $A$ of the original PDE generates the Tikhonov functional. Next, we specify our method for four (4) main classes of illposed Cauchy problems: Cauchy problems for elliptic PDEs, problems for hyperbolic and parabolic PDEs with the lateral Cauchy data and the initial boundary value problem for the parabolic PDE with the reversed time. In addition, we briefly outlinw in subsections 8.2, 8.3 extensions of this method to two important nonlinear inverse problems: inverse obstacle problems and coefficient inverse problems.

Unlike the current paper, the survey [56] was focused on the method, which was first proposed by Bukhgeim and Klibanov [24, 25, 43] for the (papers [25, 43, contain first full proofs). The method of [24] is based on Carleman estimates. The specific topic of the current paper was only briefly mentioned on pages 496-498 of [56]. The method of [24, 25, 43] was originally designed for proofs of uniqueness theorems for Coefficient Inverse Problems (CIPs) with single measurement data, see, e.g. some follow up works of Bukhgeim [26], Klibanov [44, 45, Klibanov and Timonov [52], a survey of Yamamoto [74], as well as sections 1.10 and 1.11 of the book of Beilina and Klibanov [9]. Later, this idea was extended to globally convergent numerical methods for CIPs, see works of the author with coauthors [13, 50, 51, 52, 57], the paper of Baudouin, de Buhan and Ervedoza [7] and subsection 8.3.

The role of Carleman estimates in our universal regularization method is that they provide convergence rates of minimizers of those Tikhonov functionals. The true reason why Carleman estimates are so helpful here is that they provide Hölder stability estimates in certain subdomains for those Cauchy problems for elliptic and parabolic PDEs, see, e.g. [36, 52, 56, 64]. In the hyperbolic case the Carleman estimate provides even the stronger Lipschitz stability estimate in the whole domain, see this section below and section 6 . It turns out that the Hölder stability estimate in a subdomain is a certain analog of the estimate of the modulus of the continuity of the inverse operator. On the other hand, it is one of classical results of the theory of ill-posed problems that an estimate of the modulus of the continuity on a compact set of the inverse operator provides the rate of convergence of minimizers of the Tikhonov functional, see, e.g. books of Beilina and Klibanov [9], Engl, Hanke and Neubauer [33], Kabanikhin [38], Lavrentiev, Romanov and Shishatskii [64] and Tikhonov, Goncharsky, Stepanov and Yagola [71].

The first Tikhonov functionals for ill-posed Cauchy problems for PDEs, which were generated by operators of those PDEs, were constructed in the pioneering work of Lattes and Lions [63]. Lattes and Lions have called their approach the "Quasi-Reversibility Method" (QRM). Their book contains examples of quite many ill-posed Cauchy problems. They have presented two versions of the QRM. In the first version, additional terms with regularization parameters in them were introduced in those PDEs. In the second version, strong formulations of those Cauchy problems were considered first, in which the fourth order operator $A^{*} A$ is involved. In the latter case, for elliptic and parabolic PDEs, weak variational formulations of equations with $A^{*} A$ were considered next. In 
the elliptic case, that variational formulation was equivalent with the minimization of a Tikhonov functional generated by the operator $A$. In the parabolic case, the original strong formulation led to an unnecessary term $(u(T), v(T))$ in the variational form, see the formula (3.6) on page 324 of [63]. Also, certain cut-off functions were used in [63], which is unnecessary. Convergence theorems were proved in [63]. Convergence rates were not established in [63] and Carleman estimates were not used.

First applications of the tool of Carleman estimates to this topic were done in papers of Klibanov and Santosa [47] and Klibanov and Malinsky [48. As a result, first convergence rates of minimizers of those Tikhonov functionals were established in these references. Both these works have considered the variational form of the Tikhonov functional. In [4] the Cauchy problem for the Laplace operator was considered, see the paper of Cao, Klibanov and Pereverzev [27] for a continuation of [47]. Since in the elliptic case the Hölder stability estimate can be proved by the Carleman estimate only in a subdomain, then Hölder-like convergence rates of minimizers in [27, 47] were established only in subdomains; also see sections 2-5 below.

The paper [48] is the first one where the Lipschitz stability estimate in the entire time cylinder was proved for the hyperbolic equation with the lateral Cauchy data, using a Carleman estimate (see Theorem 6.1 in section 6). Given the Carleman estimate, the Lipschitz stability estimate became possible basically because the hyperbolic equation can be stably solved in both directions of time: positive and negative. The Lipschitz stability estimate, in turn allows to establish Lipschitz-like convergence rates of minimizers of the corresponding Tikhonov functional in the entire time cylinder, see [48] and section 6 . There were several follow up works, which explored some modifications of the idea of [48] to prove the Lipschitz stability for the hyperbolic case. More precisely, those were works of Kazemi and Klibanov [46], Klibanov and Timonov [52], Isakov [36], Clason and Klibanov [29], Klibanov, Kuzhuget, Kabanikhin and Nechaev [54] and the survey [56]. While all these publications are about the case of the Euclidian geometry, the more general case of the Riemannian geometry was considered by Triggiani and Yao [72]. Lasiecka, Triggiani and Zhang [61, 62] have extended this technique to the case of the Schrödinger equation.

It is shown in section 7 that the original technique of [48] allows one to obtain the Lipschitz stability estimate, to construct the Tikhonov functional and to obtain the Lipschitzlike convergence rate of its minimizers for the problem of determining an initial condition of a hyperbolic PDE from boundary measurements. This problem is called nowadays "the problem of thermoacoustic tomography"; also, see more details in the paper of the author [55].

Based on the ideas of [47, 48], a universal regularization method for ill-posed Cauchy problems was developed in the book [52]. Later, it was briefly discussed in [56]. This method works for those PDEs, for which Carleman estimates are valid. On the first step of this approach such a Tikhonov functional is constructed which is generated by the unbounded operator of the corresponding PDE. On the second step the convergence rate of minimizers of that functional is established using a corresponding Carleman estimate. Unlike [63], cut-off functions are not used in this method. We now refer to papers of the author with coauthors [27, 29, 49, 53, 54, 55, which have explored this method.

As to the numerical implementation of our regularization method, it can work either with the finite difference, or with the finite element version, or with the spline formulation of that Tikhonov functional. The case of finite differences was implemented in [47, 63, in the paper of Klibanov and Rakesh [49] and in the paper of Klibanov, Kuzhuget, Ka- 
banikhin and Nechaev [54]. As to the finite elements, see, e.g. Bourgeois [15] and more details in section 8. Cao, Klibanov and Pereverzev [27] and Clason and Klibanov [29] have used cubic $B$-splines in numerical studies. In particular, in papers [29, 54] the problem of thermoacoustic tomography was numerically studied. Numerical testing has always shown a very good performance.

There are a large number of publications discussing solutions of ill-posed Cauchy problems for PDEs. Since the focus of this paper is on solving these problems via minimizations of Tikhonov functionals generated by corresponding PDE operators, the author refers here only to a few of such works. Other references can be found in, e.g. [38].

In a number of works of Kabanikhin with coauthors, which were summarized in the book [38], a variety of ill-posed Cauchy problems was solved via minimizations of various forms of the Tikhonov functional. Naturally, that form depends on a specific PDE; also, see, e.g. Kabanikhin and Shishlenin [39] and Karchevsky [42]. In [38, ?, 39] the operator $A$ generating the Tikhonov functional is the one, which establishes the correspondence between the sought for boundary data $q$ on an inaccessible part of the boundary and an extra boundary condition $f$ in the given Cauchy data on the accessible part of the boundary, $A q=f$. Thus, $A$ is a linear bounded operator in this case. Hao [34 as well as Hao and Lesnic [35] have published a similar approach for a parabolic equation with the lateral Cauchy data and for the Cauchy problem for the Laplace equation. Yagola, Leonov and Titarenko [73] have studied the heat equation with the reversed time and the Cauchy problem for the Laplace equation using them as some specific examples of the application of the general theory of Tikhonov functionals for linear bounded operators.

Kozlov, Maz'ya and Fomin [58] have proposed, for the first time, an alternated iterative method for the Cauchy problem for elliptic equations. This method has gained a lot of popularity since then. In this regard, we also mention the work of Avdonin, Kozlov and Maxwell [2] for a nonlinear elliptic equation and the work of Berntsson, Kozlov, Mpinganzima and Turesson [14 for the Helmholtz equation. Andrieux, Baranger and Ben Abda [1] have improved in some sense the algorithm of [58]. Lie, Hie and Zou in their elegant work [65] have constructed a version of the Adaptive Finite Element Method (adaptivity) for the Cauchy problem for an elliptic equation. As to the adaptivity for CIPs, see, e.g. [9, 12 for studies of experimental data.

Bakushinsky and Gonchasky in their book 4 , have constructed regularizing algorithms with operators $A^{*} A$ in them for solving ill-posed problems in Hilbert spaces for equations with unbounded abstract operators $A$ acting in Hilbert spaces. Also, Bakushinsky [3] has originated the method of solving ill-posed Cauchy problems for abstract operator equations in Banah spaces using finite differences with respect to one variable. In this approach the grid step size is linked with the level of error in the data, which is natural for ill-posed problems. The interest to this idea was recently renewed, see, e.g. two papers of Bakushinsky, Kokurin and Kokurin [5, 6] and references cited there. They have shown that their procedures are stable and estimated rates of convergence. Numerical results are presented in [5, 6].

Eldén [31] and then Eldén, Berntsson and Regińska [32] have proposed to solve the 1-d parabolic equation with the lateral Cauchy data on one edge of a spatial interval via considering the Fourier transform with respect to time first and then solving the Cauchy problem for the resulting PDE, using some regularization. The Hölder stability estimate was obtained in [31. Note that the technique of [32] works for spatially dependent coefficients. Furthermore, an interesting numerical example of 32 demonstrates a successful 
performance of this method on some experimental data.

The structure of this survey is the following. In section 2 we describe our universal regularization method for a generic linear PDO $A$ of the second order. Next, in sections 3-6 we illustrate how this method works for four main classes of ill-posed Cauchy problems, which are mentioned in the first paragraph of this section. In section 7 we discuss the problem of thermoacoustic tomography. Finally, in section 8 we briefly outline other results regarding the topic of this survey. Thus, an interested reader would read those results in original publications in detail. In particular, we describe in section 8 two classes of nonlinear inverse problems, to which some modifications of that universal regularization method are applicable. Those classes are: inverse obstacle problems and CIPs. All functions considered below are real valued ones.

\section{The Universal Regularization Method}

\subsection{The Carleman estimate}

We now introduce the notion of the pointwise Carleman estimate for a general Partial Differential Operator of the second order. Let $\Omega \subset \mathbb{R}^{n}$ be a bounded domain with a piecewise smooth boundary $\partial \Omega$. Let the function $\xi \in C^{2}(\bar{\Omega})$ and $|\nabla \xi| \neq 0$ in $\bar{\Omega}$. In a Carleman estimate, an important role is played by level surface of the Carleman Weight Function $(\mathrm{CWF})$. For a number $c \geq 0$ and a function $\xi(x)$ defined in $\Omega$ denote

$$
\xi_{c}=\{x \in \bar{\Omega}: \xi(x)=c\}, \Omega_{c}=\{x \in \Omega: \xi(x)>c\} .
$$

We assume that $\Omega_{c} \neq \varnothing$. Let $\Gamma_{c} \subseteq \partial \Omega, \Gamma_{c} \in C^{1}$ be a part of the boundary $\partial \Omega$ defined as $\Gamma_{c}=\{x \in \partial \Omega: \xi(x)>c\}$. We assume that $\Gamma_{c} \neq \varnothing$. Then the boundary of the domain $\Omega_{c}$ consists of two parts,

$$
\partial \Omega_{c}=\partial_{1} \Omega_{c} \cup \partial_{2} \Omega_{c}, \partial_{1} \Omega_{c}=\xi_{c}, \partial_{2} \Omega_{c}=\Gamma_{c} .
$$

Let $\lambda>1$ be a large parameter. Consider the function $\varphi_{\lambda}(x)$,

$$
\varphi_{\lambda}(x)=\exp (\lambda \xi(x)) \text {. }
$$

It follows from (2.2), (2.3) that

$$
\min _{\bar{\Omega}_{c}} \varphi_{\lambda}(x)=\left.\varphi_{\lambda}(x)\right|_{\xi_{c}}=e^{\lambda c} .
$$

Let $A(x, D)$ be a linear PDO of the second order in $\Omega$ with its principal part $A_{0}(x, D)$. We assume below that $\Gamma_{c}$ is a non-characteristic hypersurface for the operator $A_{0}$, where

$$
\begin{aligned}
A(x, D) u & =\sum_{|\alpha| \leq 2} a_{\alpha}(x) D^{\alpha} u, A_{0}(x, D) u=\sum_{|\alpha|=2} a_{\alpha}(x) D^{\alpha} u . \\
a_{\alpha} & \in C^{1}(\bar{\Omega}) \text { for }|\alpha|=2, a_{\alpha} \in C(\bar{\Omega}) \text { for }|\alpha|=0,1 .
\end{aligned}
$$

Definition 2.1. Let $\Omega_{c} \neq \varnothing$. We say that the operator $A_{0}(x, D)$ admits pointwise Carleman estimate in the domain $\Omega_{c}$ with the Carleman Weight Function $(C W F) \varphi_{\lambda}(x)$ 
if there exist constants $\lambda_{0}\left(\Omega_{c}, A_{0}\right)>1, C_{1}\left(\Omega_{c}, A_{0}\right)>0$ depending only on the domain $\Omega$ and the operator $A_{0}$, such that the following a priori estimate holds

$$
\begin{aligned}
\left(A_{0} u\right)^{2} \varphi_{\lambda}^{2}(x) & \geq C_{1} \lambda(\nabla u)^{2} \varphi_{\lambda}^{2}(x)+C_{1} \lambda^{3} u^{2} \varphi_{\lambda}^{2}(x)+\operatorname{div} U \\
\forall \lambda & \geq \lambda_{0}, \forall u \in C^{2}(\bar{\Omega}), \forall x \in \Omega_{c} .
\end{aligned}
$$

In (2.7) vector function $U(x)$ satisfies the following estimate

$$
|U(x)| \leq C_{1} \lambda^{3}\left[(\nabla u)^{2}+u^{2}\right] \varphi_{\lambda}^{2}(x), \forall x \in \Omega_{c} .
$$

Lemma 2.1. Let conditions (2.5), (2.6) hold. Suppose that the pointwise Carleman estimate (2.7)-(2.9) is valid for the principal part $A_{0}(x, D)$ of the operator $A(x, D)$. Then this estimate is also valid for the operator $A(x, D)$, although with a different constant $\lambda_{0}$. In other words, the Carleman estimate depends only on the principal part of the operator.

Proof. This lemma is elementary and well known. We have

$$
(A u)^{2} \varphi_{\lambda}^{2}(x) \geq\left(A_{0} u\right)^{2} \varphi_{\lambda}^{2}(x)-M\left[(\nabla u)^{2}+u^{2}\right] \varphi_{\lambda}^{2}(x), \forall x \in \Omega_{c}
$$

where $M>0$ is a constant depending only on the maximum of norms $\left\|a_{\alpha}\right\|_{C(\bar{\Omega})},|\alpha|=0,1$. Comparing (2.10) with (2.7) and taking $\lambda$ sufficiently large, we obtain such an analog of (2.7) in which $A_{0} u$ is replaced with $A u$.

\section{$2.2 \quad$ Hölder stability}

Consider the following Cauchy problem for the differential inequality

$$
\begin{aligned}
\left|A_{0} u\right| \leq & B(|\nabla u|+|u|+|f|) \text { in } \Omega_{c}, u \in H^{2}\left(\Omega_{c}\right), \\
u & \left|\Gamma_{c}=g_{0}(x), \partial_{n} u\right|_{\Gamma_{c}}=g_{1}(x),
\end{aligned}
$$

where $B=$ const. $>0$ and the function $f \in L_{2}\left(\Omega_{c}\right)$. Since $\Gamma_{c}$ is a non-characteristic hypersurface for the operator $A_{0}$, then functions $g_{0}, g_{1}$ in (2.12) are the Cauchy data for the function $u$. Obviously, equation $A u=f$ with the boundary data (2.12) can be reduced to the problem (2.11), (2.12). We now estimate the function $u$ via functions $f, g_{0}, g_{1}$. Such estimates were derived for parabolic, elliptic and hyperbolic operators in Chapter 4 of the book of Lavrent'ev, Romanov and Shishatskii [64], in section 2.3 of [52] and in [56].

Theorem 2.1 (Hölder stability estimate). Assume that conditions (2.5), (2.6) hold and that the Carleman estimate of Definition 2.1 is valid. Suppose that there exists a sufficiently small number $\varepsilon>0$ such that $\Omega_{c+3 \varepsilon} \neq \varnothing$ and $\Gamma_{c+3 \varepsilon} \neq \varnothing$. In addition, assume that $g_{0} \in H^{1}\left(\Gamma_{c}\right), g_{1} \in L_{2}\left(\Gamma_{c}\right), f \in L_{2}\left(\Omega_{c}\right)$. Let $m=\max _{\bar{\Omega}_{c}} \xi(x)$ and $\beta=(2 \varepsilon) /(3 m+2 \varepsilon) \in$ $(0,1)$. Assume that the function $u \in H^{2}\left(\Omega_{c}\right)$ satisfies conditions (2.11), (2.12). Then there exists a sufficiently small number $\delta_{0}=\delta_{0}\left(\varepsilon, m, B, A_{0}, \Omega_{c}\right) \in(0,1)$ and a constant $C_{2}=C_{2}\left(\varepsilon, m, B, A_{0}, \Omega_{c}\right)>0$ such that if for $\delta \in\left(0, \delta_{0}\right)$

$$
\|f\|_{L_{2}\left(\Omega_{c}\right)},\left\|g_{0}\right\|_{H^{1}\left(\Gamma_{c}\right)},\left\|g_{1}\right\|_{L_{2}\left(\Gamma_{c}\right)} \leq \delta
$$

then the following Hölder stability estimate is valid

$$
\|u\|_{H^{1}\left(\Omega_{c+3 \varepsilon}\right)} \leq C_{2}\left(1+\|u\|_{H^{1}\left(\Omega_{c}\right)}\right) \delta^{\beta}, \forall \delta \in\left(0, \delta_{0}\right) .
$$


Proof. In this proof, $C_{1}=C_{1}\left(\Omega_{c}, A_{0}\right)$ and $C_{2}=C_{2}\left(\varepsilon, m, B, A_{0}, \Omega_{c}\right)$ denote different positive constants depending on listed parameters. Since $\Omega_{c+3 \varepsilon} \neq \varnothing$ and $\Omega_{c+3 \varepsilon} \subset \Omega_{c+2 \varepsilon} \subset$ $\Omega_{c+\varepsilon} \subset \Omega_{c}$, then $\Omega_{c+2 \varepsilon}, \Omega_{c+\varepsilon}, \Omega_{c} \neq \varnothing$. Obviously $\bar{\Omega}_{c+3 \varepsilon} \cap \partial \Omega=\Gamma_{c+3 \varepsilon} \subset \Gamma_{c}$. Recall that $\Gamma_{c+3 \varepsilon} \neq \varnothing$. Choose the function $\chi(x)$ such that

$$
\chi \in C^{2}\left(\bar{\Omega}_{c}\right), \chi(x)=\left\{\begin{array}{c}
1, x \in \Omega_{c+2 \varepsilon} \\
0, x \in \Omega_{c} \backslash \Omega_{c+\varepsilon} \\
\in[0,1], x \in \Omega_{c+\varepsilon} \backslash \Omega_{c+2 \varepsilon} .
\end{array}\right.
$$

The existence of such functions is well known from the Real Analysis course. First, let $u \in C^{2}(\bar{\Omega})$. Consider the function $v=\chi u$. Representing $u=\chi u+(1-\chi) u=v+(1-\chi) u$ and using (2.11), (2.12) and (2.15), we obtain

$$
\begin{gathered}
\left|A_{0} v\right| \leq C_{2}\left[|\nabla v|+|v|+\sum_{|\alpha| \leq 2}\left|D^{\alpha}(1-\chi) u\right|+|f|\right], \forall x \in \Omega_{c} \\
\left.v\right|_{\Gamma_{c}}=\chi g_{0},\left.\partial_{n} v\right|_{\Gamma_{c}}=g_{0} \partial_{n} \chi+\chi g_{1}, \\
v(x)=0, x \in \Omega_{c} \backslash \Omega_{c+\varepsilon} .
\end{gathered}
$$

Square both sides of (2.16), multiply by $\varphi_{\lambda}^{2}(x)$ and apply (2.7). We obtain for all $\lambda>\lambda_{0}$ and all $x \in \Omega_{c}$

$$
\begin{gathered}
C_{2} f^{2} \varphi_{\lambda}^{2}(x)+C_{2} \sum_{|\alpha| \leq 2}\left|D^{\alpha}(1-\chi) u\right|^{2} \varphi_{\lambda}^{2}(x)-\operatorname{div} U \\
\geq C_{1} \lambda\left(1-\frac{C_{1}}{\lambda}\right)(\nabla v)^{2} \varphi_{\lambda}^{2}(x)+C_{1} \lambda^{3}\left(1-\frac{C_{1}}{\lambda^{3}}\right) v^{2} \varphi_{\lambda}^{2}(x) .
\end{gathered}
$$

Let $\lambda>\lambda_{1}:=\max \left(\lambda_{0}, 2 C_{1}\right)$. Then $C_{1} / \lambda<1 / 2$. Then with a different constant $C_{1}$

$$
\begin{aligned}
& C_{2} f^{2} \varphi_{\lambda}^{2}(x)+C_{2} \sum_{|\alpha| \leq 2}\left|D^{\alpha}(1-\chi) u\right|^{2} \varphi_{\lambda}^{2}(x)-\operatorname{div} U \\
\geq & C_{1} \lambda(\nabla u)^{2} \varphi_{\lambda}^{2}(x)+C_{1} \lambda^{3} u^{2} \varphi_{\lambda}^{2}(x), \\
\forall \lambda> & \lambda_{1}, \forall x \in \Omega_{c} .
\end{aligned}
$$

Integrate this inequality over $\Omega_{c}$ using Gauss' formula as well as (2.2), (2.4), (2.9), (2.15), (2.17) and (2.18). We obtain

$$
\begin{aligned}
& C_{2} e^{2 \lambda m} \int_{\Omega_{c}} f^{2} d x+C_{2} \lambda^{3} e^{2 \lambda m} \int_{\Gamma_{c}}\left[\left(\nabla g_{0}\right)^{2}+g_{1}^{2}\right] d S_{x}+C_{2} \exp [2 \lambda(c+2 \varepsilon)]\|u\|_{H^{2}(\Omega)}^{2} \\
\geq & \lambda \int_{\Omega_{c}}(\nabla v)^{2} \varphi_{\lambda}^{2} d x+\lambda^{3} \int_{\Omega_{c}} v^{2} \varphi_{\lambda}^{2} d x .
\end{aligned}
$$

Since $\Omega_{c+3 \varepsilon} \subset \Omega_{c+2 \varepsilon} \subset \Omega_{c}$, then strengthening inequality (2.19) and using (2.15), we obtain

$$
\begin{aligned}
& C_{2} e^{2 \lambda m} \int_{\Omega_{c}} f^{2} d x+C_{2} \lambda^{3} e^{2 \lambda m} \int_{\Gamma_{c}}\left[\left(\nabla g_{0}\right)^{2}+g_{1}^{2}\right] d S_{x}+C_{2} \exp [2 \lambda(c+2 \varepsilon)]\|u\|_{H^{2}(\Omega)}^{2} \\
\geq & \lambda \exp [2 \lambda(c+3 \varepsilon)] \int_{\Omega_{c+3 \varepsilon}}\left[(\nabla u)^{2}+u^{2}\right] d x .
\end{aligned}
$$


Using density arguments, we can relax now the $C^{2}$-smoothness of the function $u$ and can claim that this inequality is also valid for $u \in H^{2}(\Omega)$. Dividing both sides of this inequality by $\lambda \exp [2 \lambda(c+3 \varepsilon)]$, we conclude that there exists a number

$\lambda_{2}=\lambda_{2}\left(\varepsilon, m, B, A_{0}, \Omega_{c}\right)>\lambda_{1}$ such that for all $\lambda>\lambda_{2}$

$$
\|u\|_{H^{1}\left(\Omega_{c+3 \varepsilon}\right)}^{2} \leq C_{2} \exp [-2 \lambda \varepsilon]\|u\|_{H^{2}\left(\Omega_{c}\right)}^{2}+C_{2}\left(\left\|g_{0}\right\|_{H^{1}\left(\Gamma_{c}\right)}^{2}+\left\|g_{1}\right\|_{L_{2}\left(\Gamma_{c}\right)}^{2}+\|f\|_{L_{2}\left(\Omega_{c}\right)}^{2}\right) e^{3 \lambda m} \text {. }
$$

Hence, using (2.13), we obtain

$$
\|u\|_{H^{1}\left(G_{c+2 \varepsilon}\right)}^{2} \leq C_{2}\left(\delta^{2} e^{3 \lambda m}+e^{-2 \lambda \varepsilon}\|u\|_{H^{2}\left(\Omega_{c}\right)}^{2}\right) .
$$

We now balance two terms in the right hand side of (2.20) via choosing $\lambda=\lambda(\delta)$ such that $\delta^{2} e^{3 \lambda m}=e^{-2 \lambda \varepsilon}$. Hence,

$$
\lambda=\ln \left(\delta^{-2(3 m+2 \varepsilon)^{-1}}\right) .
$$

Hence, we should have $\delta \in\left(0, \delta_{0}\right)$, where the number $\delta_{0}=\delta_{0}\left(\varepsilon, m, B, A_{0}, \Omega_{c}\right)$ is so small that

$\ln \left(\delta_{0}^{-2(3 m+2 \varepsilon)^{-1}}\right)>\lambda_{2}$. The target estimate (2.14) follows from (2.20) and (2.21) .

Theorem 2.2 (uniqueness). Let conditions of Theorem 2.1 hold, in (2.12) $g_{0}(x) \equiv$ $g_{1}(x) \equiv 0, x \in \Gamma_{c}$ and also $f(x) \equiv 0$. Then $u(x) \equiv 0$ for $x \in \Omega_{c}$.

This theorem immediately follows from Theorem 2.1. To prove convergence of minimizers of the Tikhonov functional to the correct solution (subsection 2.3), we need to replace the pointwise inequality (2.11) with the following integral inequality

$$
\left\|A u_{\delta}\right\|_{L_{2}\left(\Omega_{c}\right)}^{2} \leq K \delta^{2}, K=\text { const. } \geq 1 .
$$

Theorem 2.3 (Hölder stability estimate). Let the $\delta$-dependent family of functions $u_{\delta} \in H^{2}\left(\Omega_{c}\right)$ satisfies inequality (2.22) with the constant $K$ independent on $\delta$. Assume that each function $u_{\delta}$ has zero boundary conditions (2.12) and that the Carleman estimate of Definition 2.1 is valid. Suppose that there exists a sufficiently small number $\varepsilon>0$ such that $\Omega_{c+3 \varepsilon} \neq \varnothing$ and $\Gamma_{c+3 \varepsilon} \neq \varnothing$. Then for the same numbers $m, \beta$ as in Theorem 2.1 there exists a sufficiently small number $\delta_{0}=\delta_{0}\left(\varepsilon, m, A, \Omega_{c}, K\right) \in(0,1)$ and a constant $C_{3}=C_{3}\left(\varepsilon, m, A, \Omega_{c}, K\right)>0$ such that for all $\delta \in\left(0, \delta_{0}\right)$ the following Hölder stability estimate holds

$$
\left\|u_{\delta}\right\|_{H^{1}\left(\Omega_{c+3 \varepsilon}\right)} \leq C_{3}\left(1+\left\|u_{\delta}\right\|_{H^{2}\left(\Omega_{c}\right)}\right) \delta^{\beta}, \forall \delta \in\left(0, \delta_{0}\right) .
$$

Proof. In this proof $C_{3}=C_{3}\left(\varepsilon, m, A, \Omega_{c}, K\right)>0$ denotes different positive constants depending on listed parameters. Assume first that the function $u \in C^{2}\left(\bar{\Omega}_{c}\right)$. We have

$$
K \delta^{2} e^{2 \lambda m} \geq \int_{\Omega_{c}}(A u)^{2} \varphi_{\lambda}^{2}(x) d x \geq \int_{\Omega_{c}}\left(A_{0} u\right)^{2} \varphi_{\lambda}^{2}(x) d x-C_{3} \int_{\Omega_{c}}\left((\nabla u)^{2}+u^{2}\right) \varphi_{\lambda}^{2}(x) d x .
$$

This is equivalent with

$$
K \delta^{2} e^{2 \lambda m}+C_{3} \int_{\Omega_{c}}\left((\nabla u)^{2}+u^{2}\right) \varphi_{\lambda}^{2}(x) d x \geq \int_{\Omega_{c}}\left(A_{0} u\right)^{2} \varphi_{\lambda}^{2}(x) d x .
$$

The rest of the proof is similar with the proof of Theorem 2.1. The replacement of $u \in C^{2}\left(\bar{\Omega}_{c}\right)$ with $u \in H^{2}\left(\Omega_{c}\right)$ is done using density arguments. 


\subsection{Regularization}

Cauchy Problem. Find the function $u \in H^{2}\left(\Omega_{c}\right)$ satisfying the following conditions

$$
\begin{aligned}
A u & =f \text { in } \Omega_{c}, \\
u & \left|\Gamma_{c}=g_{0}(x), \partial_{n} u\right|_{\Gamma_{c}}=g_{1}(x) .
\end{aligned}
$$

Assume that there exists a function $F \in H^{2}\left(\Omega_{c}\right)$ such that

$$
\left.F\right|_{\Gamma_{c}}=g_{0}(x),\left.\partial_{n} F\right|_{\Gamma_{c}}=g_{1}(x) \text {. }
$$

We find an approximate solution of the problem (2.23), (2.24) as a minimizer of the following Tikhonov functional with the regularization parameter $\gamma \in(0,1)$,

$$
\begin{aligned}
J_{\gamma}(u)= & \|A u-f\|_{L_{2}\left(\Omega_{c}\right)}^{2}+\gamma\|u-F\|_{H^{2}\left(\Omega_{c}\right)}^{2}, u \in H^{2}\left(\Omega_{c}\right), \\
& \text { subject to the Cauchy boundary data }(\underline{2.24}) .
\end{aligned}
$$

In the regularization theory, such a minimizer is called regularized solution, see, e.g. [9, 71]. Thus, we regularize the problem (2.23), (2.24), which, at least in general, is illposed. First, we prove the existence and uniqueness of the minimizer of the functional (2.26) with conditions (2.27).

Theorem 2.4 (existence). For every $\gamma \in(0,1)$ there exists unique minimizer $u_{\gamma} \in$ $H^{2}\left(\Omega_{c}\right)$ of the functional $J_{\gamma}(u)$ and with a constant $C_{4}=C_{4}\left(\Omega_{c}, A\right)>0$ the following estimate holds

$$
\left\|u_{\gamma}\right\|_{H^{2}\left(\Omega_{c}\right)} \leq \frac{C_{4}}{\sqrt{\gamma}}\left(\|F\|_{H^{2}\left(\Omega_{c}\right)}+\|f\|_{L_{2}\left(\Omega_{c}\right)}\right) .
$$

.Proof. In this proof $C_{4}=C_{4}\left(\Omega_{c}, A\right)>0$ denotes different constant depending on listed parameters. Denote

$$
H_{0, c}^{2}\left(\Omega_{c}\right)=\left\{v \in H^{2}\left(\Omega_{c}\right):\left.v\right|_{\Gamma_{c}}=\left.\partial_{n} v\right|_{\Gamma_{c}}=0\right\} .
$$

Let $v=u-F$. Then $v \in H_{0, c}^{2}\left(\Omega_{c}\right)$.By (2.25), (2.26) and (2.27) we should minimize the following functional $\bar{J}_{\gamma}(v)$

$$
\bar{J}_{\gamma}(v)=\|A v+(A F-f)\|_{L_{2}\left(\Omega_{c}\right)}^{2}+\gamma\|v\|_{H^{2}\left(\Omega_{c}\right)}^{2}, v \in H_{0, c}^{2}\left(\Omega_{c}\right) .
$$

If $v_{\gamma} \in H_{0, c}^{2}\left(\Omega_{c}\right)$ is a minimizer of the functional (2.29), then $u_{\gamma}=v_{\gamma}+F$ is a minimizer of the functional (2.26) satisfying conditions (2.27). And vice versa: if $u_{\gamma}$ is a minimizer of the functional (2.26) satisfying conditions (2.27), then $v_{\gamma}=u_{\gamma}-F \in H_{0, c}^{2}\left(\Omega_{c}\right)$ is a minimizer of the functional (2.29).

By the variational principle any minimizer $v_{\gamma}$ of the functional (2.29) should satisfy the following condition

$$
\left(A v_{\gamma}, A h\right)+\gamma\left[v_{\gamma}, h\right]=(A h, f-A F), \forall h \in H_{0, c}^{2}\left(\Omega_{c}\right),
$$

where $($,$) and [,] are scalar products in L_{2}\left(\Omega_{c}\right)$ and $H^{2}\left(\Omega_{c}\right)$ respectively. Denote

$$
\{v, h\}_{\gamma}=(A v, A h)+\gamma[v, h], \forall v, h \in H_{0, c}^{2}\left(\Omega_{c}\right) .
$$


Hence, $\{v, h\}$ defines a new scalar product in the Hilbert space $H_{0}^{2}\left(\Omega_{c}\right)$ and the corresponding norm $\{v\}$ satisfies

$$
\sqrt{\gamma}\|v\|_{H^{2}\left(\Omega_{c}\right)} \leq\{v\}_{\gamma} \leq C_{4}\|v\|_{H^{2}\left(\Omega_{c}\right)} .
$$

Thus, the scalar product (2.31) generates the new norm $\{v\}_{\gamma}$, which is equivalent with the norm $\|v\|_{H^{2}\left(\Omega_{c}\right)}$. Hence, (2.30) can be rewritten as

$$
\left\{v_{\gamma}, h\right\}_{\gamma}=(A h, f-A F), \forall h \in H_{0, c}^{2}\left(\Omega_{c}\right) .
$$

It follows from (2.32) that

$$
|(A h, f-A F)| \leq C_{4}\left(\|F\|_{H^{2}\left(\Omega_{c}\right)}+\|f\|_{L_{2}\left(\Omega_{c}\right)}\right)\{h\}_{\gamma} .
$$

Hence, the right hand side of (2.33) can be considered as a bounded linear functional defined on the space $H_{0}^{2}\left(\Omega_{c}\right)$. Hence, by Riesz theorem there exists an element $w_{\gamma}=$ $w_{\gamma}(f-A F)$ such that $(A h, f-A F)=\left\{w_{\gamma}, h\right\}_{\gamma}, \forall h \in H_{0, c}^{2}\left(\Omega_{c}\right)$. This and (2.33) imply that $\left\{v_{\gamma}, h\right\}_{\gamma}=\left\{w_{\gamma}, h\right\}_{\gamma}, \forall h \in H_{0, c}^{2}\left(\Omega_{c}\right)$. Hence, the minimizer $v_{\gamma}$ exists and $v_{\gamma}=w_{\gamma}$. Also, by Riesz theorem and (2.34) $\left\{v_{\gamma}\right\}_{\gamma} \leq C_{4}\left(\|F\|_{H^{2}\left(\Omega_{c}\right)}+\|f\|_{L_{2}\left(\Omega_{c}\right)}\right)$. Hence, the minimizer $v_{\gamma}$ is unique and the left inequality (2.32) implies (2.28).

In the proof of Theorem 2.4 we have used only the variational principle and Riesz theorem. However, the Carleman estimate (2.7) was not used. We use this estimate in Theorem 2.5, which establishes the convergence rate of minimizers $u_{\gamma}$ to the exact solution, under suitable conditions. Note that convergence is established in a subdomain $\Omega_{c+3 \varepsilon} \subset \Omega_{c}$, which is a little bit less than the original domain $\Omega_{c}$. Contrary to this, we show in section 6 that for the hyperbolic case convergence takes place in the whole domain of interest, which is actually the time cylinder in that case.

Following one of concepts of Tikhonov (see, e.g. section 1.4 of [9]), we assume that there exists an exact solution $u^{*}$ of the problem (2.23), (2.24) with the exact data $f^{*} \in$ $L_{2}\left(\Omega_{c}\right),\left.u^{*}\right|_{\Gamma_{c}}=g_{0}^{*} \in H^{1}\left(\Gamma_{c}\right),\left.\partial_{n} u^{*}\right|_{\Gamma_{c}}=g_{1}^{*} \in L_{2}\left(\Gamma_{c}\right)$. By Theorem 2.2 the exact solution $u^{*}$ is unique. Because of the existence of $u^{*}$, there also exists an exact function $F^{*} \in$ $H^{2}\left(\Omega_{c}\right)$ satisfying boundary conditions (2.25), in which functions $g_{0}, g_{1}$ are replaced with functions $g_{0}^{*}, g_{1}^{*}$. Here is an example of such a function $F^{*}$. Let the function $\rho \in C^{2}\left(\bar{\Omega}_{c}\right)$ be such that $\rho(x)=1$ in a small neighborhood $N_{\sigma}\left(\Gamma_{c}\right)=\left\{x \in \Omega_{c}: \operatorname{dist}\left(x, \Gamma_{c}\right)<\sigma\right\}$ and $\rho(x)=0$ for $x \in \Omega_{c} \backslash N_{2 \sigma}\left(\Gamma_{c}\right)$, where $\sigma>0$ is a sufficiently small number. Then $F^{*}$ can be constructed as $F^{*}(x)=\rho(x) u^{*}(x)$. Let $\delta>0$ be a sufficiently small number characterizing the error in the data. We assume that

$$
\left\|f^{*}-f\right\|_{L_{2}\left(\Omega_{c}\right)},\left\|g_{0}^{*}-g_{0}\right\|_{H^{1}\left(\Gamma_{c}\right)},\left\|g_{1}^{*}-g_{1}\right\|_{L_{2}\left(\Gamma_{c}\right)} \leq \delta,\left\|F^{*}-F\right\|_{H^{2}\left(\Omega_{c}\right)} \leq \delta
$$

Theorem 2.5 (convergence rate). Assume that the Carleman estimate of Definition 2.1 holds, conditions (2.25) and (2.35) are valid and let the regularization parameter $\gamma=\gamma(\delta)=\delta^{2 \alpha}$, where $\alpha=$ const. $\in(0,1]$. Suppose that there exists a sufficiently small number $\varepsilon>0$ such that $\Omega_{c+3 \varepsilon} \neq \varnothing$ and $\Gamma_{c+3 \varepsilon} \neq \varnothing$. Let numbers $m, \beta$ be the same as in Theorem 2.1. Then there exists a sufficiently small number $\delta_{0}=\delta_{0}\left(\varepsilon, m, A, \Omega_{c}\right) \in(0,1)$ and a constant $C_{5}=C_{5}\left(\varepsilon, m, A, \Omega_{c}\right)>0$ such that if $\delta \in\left(0, \delta_{0}^{1 / \alpha}\right)$, then the following convergence rate is valid

$$
\left\|u_{\gamma}-u^{*}\right\|_{H^{1}\left(\Omega_{c+3 \varepsilon}\right)} \leq C_{5}\left(1+\left\|u^{*}\right\|_{H^{2}\left(\Omega_{c}\right)}\right) \delta^{\alpha \beta}, \forall \delta \in\left(0, \delta_{0}\right),
$$


where $u_{\gamma(\delta)}$ is the minimizer of the functional (2.26), (2.27) which is guaranteed by Theorem 2.4.

Proof. In this proof $C_{5}=C_{5}\left(\varepsilon, m, A, \Omega_{c}\right)>0$ denotes different positive constants depending on listed parameters. Let $v^{*}=u^{*}-F^{*}$. Then $v^{*} \in H_{0, c}^{2}\left(\Omega_{c}\right)$ and $A v^{*}=$ $f^{*}-A F^{*}$. Hence,

$$
\left(A v^{*}, A h\right)+\gamma\left[v^{*}, h\right]=\left(A h, f^{*}-A F^{*}\right)+\gamma\left[v^{*}, h\right], \forall h \in H_{0}^{2}\left(\Omega_{c}\right) .
$$

Subtract identity (2.30) from identity (2.37) and denote $\widetilde{v}_{\gamma}=v^{*}-v_{\gamma}, \widetilde{f}=f^{*}-f, \widetilde{F}=$ $F^{*}-F$. Then

$$
\left(A \widetilde{v}_{\gamma}, A h\right)+\gamma\left[\widetilde{v}_{\gamma}, h\right]=(A h, \widetilde{f}-A \widetilde{F})+\gamma\left[v^{*}, h\right], \forall h \in H_{0}^{2}\left(\Omega_{c}\right) .
$$

Setting here $h:=\widetilde{v}_{\gamma}$, we obtain

$$
\left\|A \widetilde{v}_{\gamma}\right\|_{L_{2}\left(\Omega_{c}\right)}^{2}+\gamma\left\|\widetilde{v}_{\gamma}\right\|_{H^{2}\left(\Omega_{c}\right)}^{2}=\left(A \widetilde{v}_{\gamma}, \widetilde{f}-A \widetilde{F}\right)+\gamma\left[v^{*}, \widetilde{v}_{\gamma}\right]
$$

Applying the Cauchy-Schwarz inequality to (2.38), we obtain

$$
\begin{gathered}
\left\|A \widetilde{v}_{\gamma}\right\|_{L_{2}\left(\Omega_{c}\right)}^{2}+\gamma\left\|\widetilde{v}_{\gamma}\right\|_{H^{2}\left(\Omega_{c}\right)}^{2} \\
\leq \frac{1}{2}\left\|A \widetilde{v}_{\gamma}\right\|_{L_{2}\left(\Omega_{c}\right)}^{2}+\frac{1}{2}\|\widetilde{f}-A \widetilde{F}\|_{L_{2}\left(\Omega_{c}\right)}^{2}+\frac{\gamma}{2}\left\|v^{*}\right\|_{H^{2}\left(\Omega_{c}\right)}^{2}+\frac{\gamma}{2}\left\|\widetilde{v}_{\gamma}\right\|_{H^{2}\left(\Omega_{c}\right)}^{2} .
\end{gathered}
$$

Hence, by (2.35)

$$
\left\|A \widetilde{v}_{\gamma}\right\|_{L_{2}\left(\Omega_{c}\right)}^{2}+\gamma\left\|\widetilde{v}_{\gamma}\right\|_{H^{2}\left(\Omega_{c}\right)}^{2} \leq C_{5} \delta^{2}+\gamma\left\|v^{*}\right\|_{H^{2}\left(\Omega_{c}\right)}^{2}
$$

Since $\gamma=\delta^{2 \alpha}$, where $\alpha \in(0,1]$, then $\delta^{2} \leq \gamma$. Hence, (2.40) implies that

$$
\begin{gathered}
\left\|\widetilde{v}_{\gamma}\right\|_{H^{2}\left(\Omega_{c}\right)} \leq C_{5}\left(1+\left\|v^{*}\right\|_{H^{2}\left(\Omega_{c}\right)}\right), \\
\left\|A \widetilde{v}_{\gamma}\right\|_{L_{2}\left(\Omega_{c}\right)}^{2} \leq C_{5}\left(1+\left\|v^{*}\right\|_{H^{2}\left(\Omega_{c}\right)}^{2}\right) \delta^{2 \alpha} .
\end{gathered}
$$

Let $w_{\gamma}=\widetilde{v}_{\gamma}\left(1+\left\|v^{*}\right\|_{H^{2}\left(\Omega_{c}\right)}\right)^{-1}$. Then (2.41), (2.42) and Theorem 2.3 imply that $\left\|w_{\gamma}\right\|_{H^{1}\left(\Omega_{c+3 \varepsilon}\right)} \leq C_{5} \delta^{\alpha \beta}, \forall \delta \in\left(0, \delta_{0}\right)$. Therefore,

$$
\left\|\widetilde{v}_{\gamma}\right\|_{H^{1}\left(\Omega_{c+3 \varepsilon}\right)} \leq C_{5}\left(1+\left\|v^{*}\right\|_{H^{2}\left(\Omega_{c}\right)}\right) \delta^{\alpha \beta}, \forall \delta \in\left(0, \delta_{0}\right) .
$$

Next, since $\widetilde{v}_{\gamma}=\left(u_{\gamma}-u^{*}\right)+\left(F^{*}-F\right)$ and since by (2.35) $\left\|F^{*}-F\right\|_{H^{1}\left(\Omega_{c+3 \varepsilon}\right)} \leq \delta$, then the triangle inequality implies that

$$
\left\|\widetilde{v}_{\gamma}\right\|_{H^{1}\left(\Omega_{c+3 \varepsilon}\right)} \geq\left\|u_{\gamma}-u^{*}\right\|_{H^{1}\left(\Omega_{c+3 \varepsilon}\right)}-\left\|F^{*}-F\right\|_{H^{1}\left(\Omega_{c+3 \varepsilon}\right)} \geq\left\|u_{\gamma}-u^{*}\right\|_{H^{1}\left(\Omega_{c+3 \varepsilon}\right)}-\delta .
$$

Since numbers $\beta, \delta \in(0,1)$ and since $\alpha \in(0,1]$, then $\delta^{\alpha \beta}>\delta$. Thus, using (2.43) and (2.44), we obtain (2.36) . 


\section{Cauchy Problem for the Elliptic Equation}

We now rewrite the operator $A$ in (2.5) as

$$
\begin{aligned}
L u & =\sum_{i, j=1}^{n} a_{i, j}(x) u_{x_{i} x_{j}}+\sum_{j=1}^{n} b_{j}(x) u_{x_{j}}+b_{0}(x) u, x \in \Omega, \\
L_{0} u & =\sum_{i, j=1}^{n} a_{i, j}(x) u_{x_{i} x_{j}}
\end{aligned}
$$

where $a_{i, j}(x)=a_{j, i}(x), \forall i, j$ and $L_{0}$ is the principal part of the operator $L$. As in (2.6), we assume that

$$
a_{i, j} \in C^{1}(\bar{\Omega}) ; b_{j}, b_{0} \in C(\bar{\Omega}) .
$$

The ellipticity of the operator $L$ deals only with its principal part $L_{0}$ and it means that there exist two constants $\mu_{1}, \mu_{2}>0, \mu_{1} \leq \mu_{2}$ such that

$$
\mu_{1}|\eta|^{2} \leq \sum_{i, j=1}^{n} a_{i, j}(x) \eta_{i} \eta_{j} \leq \mu_{2}|\eta|^{2}, \forall x \in \bar{\Omega}, \forall \eta=\left(\eta_{1}, \ldots \eta_{n}\right) \in \mathbb{R}^{n}
$$

Let $\Theta \subset \partial \Omega$ be the part of the boundary $\partial \Omega$, where the Cauchy data are given. Assume that the equation of $\Theta$ is $\Theta=\left\{x \in \mathbb{R}^{n}: x_{1}=g\left(x_{2}, \ldots, x_{n}\right),\left(x_{2}, \ldots, x_{n}\right) \in \Theta^{\prime} \subset \mathbb{R}^{n-1}\right\}$ and that the function $g \in C^{2}\left(\bar{\Theta}^{\prime}\right)$. Here $\Theta^{\prime} \subset \mathbb{R}^{n-1}$ is a bounded domain. Changing variables as $x=\left(x_{1}, x_{2}, \ldots, x_{n}\right) \Leftrightarrow\left(x_{1}^{\prime}, x_{2}, \ldots, x_{n}\right)$, where $x_{1}^{\prime}=x_{1}-g\left(x_{2}, \ldots, x_{n}\right)$, we obtain that in new variables

$\Theta=\left\{x \in \mathbb{R}^{n}: x_{1}=0,\left(x_{2}, \ldots, x_{n}\right) \in \Theta^{\prime}\right\}$. Here we kept the same notation for $x_{1}$ as before: for the simplicity of notations. This change of variables does not affect the property of the ellipticity of the operator $L$. Let $X>0$ be a certain number. Denote $\bar{x}=\left(x_{2}, \ldots, x_{n}\right)$. Thus, without any loss of generality, we assume that

$$
\Omega \subset\left\{x_{1}>0\right\}, \Theta=\left\{x \in \mathbb{R}^{n}: x_{1}=0,|\bar{x}|<X\right\} \subset \partial \Omega .
$$

Let the function $f \in L_{2}(\Omega)$. Consider the elliptic equation,

$$
L u=f \text { in } \Omega .
$$

Cauchy Problem for the Elliptic Equation. Let the part $\Theta$ of the boundary $\partial \Omega$ be given by (3.5). Find such a function $u \in H^{2}(\Omega)$ that satisfies equation (3.6) and has the following Cauchy data $g_{0}, g_{1}$ at $\Theta$

$$
\left.u\right|_{\Theta}=g_{0}(\bar{x}),\left.u_{x_{1}}\right|_{\Theta}=g_{1}(\bar{x}) .
$$

These are incomplete Cauchy data, since they are given only at a part of the boundary of the domain $\Omega$ rather than at the whole boundary. Let $\lambda>1$ and $\nu>1$ be two large parameters, which we define later. Consider two arbitrary numbers $a, c=$ const. $\in(0,1)$, where $a<c$. To introduce the Carleman estimate, consider functions $\psi(x), \varphi_{\lambda}(x)$ defined as

$$
\psi(x)=x_{1}+\frac{|\bar{x}|^{2}}{X^{2}}+a, \varphi_{\lambda}(x)=\exp \left(\lambda \psi^{-\nu}\right) .
$$


Then the analogs of (2.1) and $\Gamma_{c}$ are

$$
\begin{aligned}
\Omega_{c} & =\left\{x: x_{1}>0, x_{1}+\frac{|\bar{x}|^{2}}{X^{2}}+a<c\right\}, \xi_{c}=\left\{x: x_{1}>0, x_{1}+\frac{|\bar{x}|^{2}}{X^{2}}+a=c\right\}, \\
\Gamma_{c} & =\left\{x: x_{1}=0,|\bar{x}|<\sqrt{c-a} X\right\} \\
\partial \Omega_{c} & =\xi_{c} \cup \Gamma_{c} .
\end{aligned}
$$

We assume that $\bar{\Omega}_{c} \subseteq \Omega$. By (3.5) and (3.9) $\Gamma_{c} \subset \Theta$. For a sufficiently small number $\varepsilon>0$ define the subdomain $\Omega_{c+3 \varepsilon} \subset \Omega_{c}$ as

$$
\Omega_{c+3 \varepsilon}=\left\{x: x_{1}>0, x_{1}+\frac{|\bar{x}|^{2}}{X^{2}}+a<c-3 \varepsilon\right\}, \varepsilon \in(0,(c-a) / 3) .
$$

Lemma 3.1 follows immediately from Lemma 3 of $\S 1$ of chapter 4 of the book [64].

Lemma 3.1 (Carleman estimate). There exist a sufficiently large number $\nu_{0}=\nu_{0}\left(a, c, \mu_{1}, \mu_{2}, \max _{i, j}\left\|a_{i, j}\right\|_{C^{1}\left(\bar{\Omega}_{c}\right)}, X\right)>1$ and a sufficiently large absolute constant $\lambda_{0}>1$ such that for all $\nu \geq \nu_{0}, \lambda \geq \lambda_{0}$ and for all functions $u \in C^{2}\left(\bar{\Omega}_{c}\right)$ the following pointwise Carleman estimate is valid for all $x \in \Omega_{c}$ with a constant $C=$ $C\left(n, \max _{i, j}\left\|a_{i, j}\right\|_{C^{1}\left(\bar{\Omega}_{c}\right)}\right)$

$$
\begin{aligned}
\left(L_{0} u\right)^{2} \varphi_{\lambda}^{2} & \geq C \lambda|\nabla u|^{2} \varphi_{\lambda}^{2}+C \lambda^{3} u^{2} \varphi_{\lambda}^{2}+\operatorname{div} U \\
|U| & \leq C \lambda^{3}\left[(\nabla u)^{2}+u^{2}\right] \varphi_{\lambda}^{2} .
\end{aligned}
$$

This Carleman estimate allows us to construct the Tikhonov functional for solving the Cauchy problem (3.6), (3.7). First, we construct an example of the function $F \in H^{2}\left(\Omega_{c}\right)$ : as in (2.25). Assume that functions

$$
g_{0}, g_{1} \in H^{2}\left(\Gamma_{c}\right)
$$

where $\Gamma_{c}$ is defined in (3.9). Even though the minimal smoothness requirement should probably be $g_{0} \in H^{1}\left(\Gamma_{c}\right), g_{1} \in L_{2}\left(\Gamma_{c}\right)$, we still assume a little bit higher smoothness (3.12) here only for the sake of our specific example of the function $F$. Let $\sigma \in$ $(0,(c-a) / 2)$ be a sufficiently small number. Consider the function $\rho\left(x_{1}\right)$ such that

$$
\rho \in C^{2}[0, c-a], \rho\left(x_{1}\right)=\left\{\begin{array}{c}
1, x_{1} \in(0, \sigma) \\
0, x_{1} \in[2 \sigma, c-a]
\end{array}\right.
$$

We construct the function $F$ as

$$
F(x)=\rho\left(x_{1}\right) g_{0}(\bar{x})-\rho\left(x_{1}\right) x_{1} g_{1}(\bar{x}) .
$$

By (3.12)-(3.14) the function $F \in H^{2}\left(\Omega_{c}\right)$ and also by (3.7)-(3.12) $\left.F\right|_{\Gamma_{c}}=g_{0}(\bar{x}),\left.\partial_{n} F\right|_{\Gamma_{c}}=$ $g_{1}(\bar{x})$, where $\partial_{n}=-\partial_{x_{1}}$. We now assume the existence of the exact solution $u^{*}$ of the problem (3.6), (3.7) with the exact Cauchy data $g_{0}^{*}, g_{1}^{*} \in H^{2}\left(\Gamma_{c}\right)$ and the exact right hand side $f^{*}$. Next, we construct the function $F^{*} \in H^{2}\left(\Omega_{c}\right)$ as above via replacing in (3.14) $g_{0}, g_{1}$ with $g_{0}^{*}, g_{1}^{*}$. 
We construct the direct analog of the Tikhonov functional (2.26) with boundary conditions (2.27) as

$$
\begin{aligned}
J_{\gamma}^{L}(u)= & \|L u-f\|_{L_{2}\left(\Omega_{c}\right)}^{2}+\gamma\|u-F\|_{H^{2}\left(\Omega_{c}\right)}^{2}, u \in H^{2}\left(\Omega_{c}\right), \\
& \text { subject to the Cauchy boundary data } g_{0}, g_{1} \text { on } \Gamma_{c},
\end{aligned}
$$

where the function $F$ is defined in (3.14). Theorem 3.1 follows immediately from Theorems 2.4 .

Theorem 3.1. For every $\gamma \in(0,1)$ there exists unique minimizer $u_{\gamma} \in H^{2}\left(\Omega_{c}\right)$ of the functional $J_{\gamma}^{L}(u)$ and with a constant $C_{6}=C_{6}\left(\Omega_{c}, L, \rho_{1}\right)>0$ the following estimate holds

$$
\left\|u_{\gamma}\right\|_{H^{2}\left(\Omega_{c}\right)} \leq \frac{C_{6}}{\sqrt{\gamma}}\left(\|F\|_{H^{2}\left(\Omega_{c}\right)}+\|f\|_{L_{2}\left(\Omega_{c}\right)}\right) .
$$

The convergence Theorem 3.2 follows immediately from Theorems 2.5, 3.1, Lemma 3.1 and (3.8)-(3.11).

Theorem 3.2 (convergence rate). Assume that conditions (3.12)-(3.14) are valid. Also, assume that conditions (2.35) are satisfied. Let in (3.15) the regularization parameter $\gamma=\gamma(\delta)=\delta^{2 \alpha}$, where $\alpha=$ const. $\in(0,1]$. Let $\varepsilon>0$ be a sufficiently small and the domain $\Omega_{c+3 \varepsilon}$ be as in (3.11). Let the number $m=a^{-\nu_{0}}$, where $\nu_{0}=$ $\nu_{0}\left(a, c, \mu_{1}, \mu_{2}, \max _{i, j}\left\|a_{i, j}\right\|_{C^{1}\left(\bar{\Omega}_{c}\right)}, X\right)>1$ is the number of Lemma 3.1. Define the number $\beta \in(0,1)$ as $\beta=(2 \varepsilon) /(3 m+2 \varepsilon)$. Then there exists a sufficiently small number $\delta_{0}=\delta_{0}\left(\varepsilon, \nu_{0}, L, \Omega_{c}, \rho_{1}\right) \in(0,1)$ and a constant $C_{7}=C_{7}\left(\varepsilon, \nu_{0}, L, \Omega_{c}, \rho_{1}\right)>0$ such that if $\delta \in\left(0, \delta_{0}^{1 / \alpha}\right)$, then the following convergence rate is valid

$$
\left\|u_{\gamma(\delta)}-u^{*}\right\|_{H^{1}\left(\Omega_{c+3 \varepsilon}\right)} \leq C_{7}\left(1+\left\|u^{*}\right\|_{H^{2}\left(\Omega_{c}\right)}\right) \delta^{\alpha \beta}, \forall \delta \in\left(0, \delta_{0}\right),
$$

where $u_{\gamma(\delta)}$ is the minimizer of the functional (3.15), (3.16), which is guaranteed by Theorem 3.1 .

\section{Parabolic Equation With the Lateral Cauchy Data}

Let $G \subset \mathbb{R}^{n}$ be a bounded domain with a piecewise smooth boundary and $T=$ const. $>0$. Denote $G_{T}=G \times(-T, T)$. Let $L_{p a r}$ be the elliptic operator of the second order in $G_{T}$, which we define the same way as the operator $L$ in (3.1)-(3.4) with the only difference that now its coefficients depend on both $x$ and $t$ and the domain $\Omega$ is replaced with the domain $G_{T}$. Let $L_{0, p a r}$ be the similarly defined principal part of the operator $L_{\text {par }}$, see (3.2). For brevity we are not rewriting (3.1)-(3.4). Next, we define the parabolic operator as $P=\partial_{t}-L_{p a r}$ and the principal part of $P$ is $P_{0}=\partial_{t}-L_{0, p a r}$. Let $\Theta \subset \partial G, \Theta \in C^{2}$ be the subsurface of the boundary $\partial G$ with the same properties as ones in section 3 . Denote $\Theta_{T}=\Theta \times(-T, T)$. Without a loss of generality we assume that for a certain number $X>0$

$$
G \subset\left\{x_{1}>0\right\}, \Theta=\left\{x \in \mathbb{R}^{n}: x_{1}=0,|\bar{x}|<X\right\} \subset \partial G .
$$

Let the function $f(x, t) \in L_{2}\left(G_{T}\right)$. Consider the parabolic equation

$$
P u:=u_{t}-L_{p a r} u=f \text { in } G_{T} .
$$


Cauchy Problem with the Lateral Data for the Parabolic Equation. Let the part $\Theta$ of the boundary $\partial G$ be given by equation (4.1). Find such a function $u \in H^{2}\left(G_{T}\right)$ that satisfies equation (4.2) and has the following lateral Cauchy data $g_{0}, g_{1}$ at $\Theta_{T}$

$$
\left.u\right|_{\Theta_{T}}=g_{0}(\bar{x}, t),\left.u_{x_{1}}\right|_{\Theta_{T}}=g_{1}(\bar{x}, t) .
$$

We are using here the smoothness $u \in H^{2}\left(G_{T}\right)$, which is a little bit higher than possibly the minimal required smoothness $u \in H^{2,1}\left(G_{T}\right)$, because we need this smoothness in Lemma 4.2. We introduce the Carleman estimate similarly with section 3 . Let $\lambda>1$ and $\nu>1$ be two large parameters, which we define later. Consider two arbitrary numbers $a, c=$ const. $\in(0,1)$, where $a<c$. Consider functions $\psi(x, t), \varphi_{\lambda}(x, t)$ defined as

$$
\psi(x, t)=x_{1}+\frac{|\bar{x}|^{2}}{X^{2}}+\frac{t^{2}}{T^{2}}+a, \varphi_{\lambda}(x, t)=\exp \left(\lambda \psi^{-\nu}\right) .
$$

Analogs of (3.8)-(3.11) are

$$
\begin{aligned}
\Omega_{c} & =\left\{(x, t): x_{1}>0, x_{1}+\frac{|\bar{x}|^{2}}{X^{2}}+\frac{t^{2}}{T^{2}}+a<c\right\} \\
\xi_{c} & =\left\{(x, t): x_{1}>0, x_{1}+\frac{|\bar{x}|^{2}}{X^{2}}+\frac{t^{2}}{T^{2}}+a=c\right\} \\
\Gamma_{c} & =\left\{(x, t): x_{1}=0, \frac{|\bar{x}|^{2}}{X^{2}}+\frac{t^{2}}{T^{2}}<c-a\right\} \\
\partial \Omega_{c} & =\xi_{c} \cup \Gamma_{c}, \\
\Omega_{c+3 \varepsilon} & =\left\{(x, t): x_{1}>0, x_{1}+\frac{|\bar{x}|^{2}}{X^{2}}+\frac{t^{2}}{T^{2}}+a<c-3 \varepsilon\right\}, \varepsilon \in(0,(c-a) / 3) .
\end{aligned}
$$

We assume that $\Omega_{c} \subset \bar{G}_{T}$. By (4.1) and (4.7) $\Gamma_{c} \subset \Theta_{T}$. Lemma 4.1 follows immediately from Lemma 3 of $\S 1$ of chapter 4 of the book [64].

Lemma 4.1 (Carleman estimate). There exist a sufficiently large number $\nu_{0}=\nu_{0}\left(a, c, \mu_{1}, \mu_{2}, \max _{i, j}\left\|a_{i, j}\right\|_{C^{1}\left(\bar{\Omega}_{c}\right)}, X, T\right)>1$ and a sufficiently large absolute constant $\lambda_{0}>1$ such that for all $\nu \geq \nu_{0}, \lambda \geq \lambda_{0}$ and for all functions $u \in C^{2,1}\left(\bar{\Omega}_{c}\right)$ the following pointwise Carleman estimate is valid for all $(x, t) \in \Omega_{c}$ with a constant $C=C\left(n, \max _{i, j}\left\|a_{i, j}\right\|_{C^{1}\left(\bar{\Omega}_{c}\right)}\right)$

$$
\begin{aligned}
\left(P_{0} u\right)^{2} \varphi_{\lambda}^{2} & \geq C \lambda|\nabla u|^{2} \varphi_{\lambda}^{2}+C \lambda^{3} u^{2} \varphi_{\lambda}^{2}+\operatorname{div} U+V_{t} \\
|U|,|V| & \leq C \lambda^{3}\left[(\nabla u)^{2}+u_{t}^{2}+u^{2}\right] \varphi_{\lambda}^{2} .
\end{aligned}
$$

Similarly with (3.12), let functions

$$
g_{0}, g_{1} \in H^{2}\left(\Gamma_{c}\right)
$$

with the same comments about a little bit higher smoothness requirements (4.9) as in lines below (3.12). In (4.9) $\Gamma_{c}$ is the same as in (4.6). Let the number $\sigma$ be the same as 
in section 3 and $\rho\left(x_{1}\right)$ be the function defined in (3.13). We now construct the function $F(x, t) \in H^{2}\left(\Omega_{c}\right)$ as

$$
F(x, t)=\rho\left(x_{1}\right) g_{0}(\bar{x}, t)-\rho\left(x_{1}\right) x_{1} g_{1}(\bar{x}, t) .
$$

By (4.6), (4.9) and (4.10) $F \in H^{2}\left(\Omega_{c}\right)$. In addition, $\left.F\right|_{\Gamma_{c}}=g_{0}(\bar{x}, t),\left.\partial_{n} F\right|_{\Gamma_{c}}=g_{1}(\bar{x}, t)$, where $\partial_{n}=-\partial_{x_{1}}$. Again, we assume the existence of the exact solution $u^{*}$ of the problem (4.2), (4.3) with the exact Cauchy data $g_{0}^{*}, g_{1}^{*}$ and with the exact right hand side $f^{*}$ and we construct the function $F^{*} \in H^{2,1}\left(\Omega_{c}\right)$ similarly.

Given the function $F$ in (4.10), the Tikhonov functional is now constructed as

$$
\begin{aligned}
J_{\gamma}(u)= & \|P u-f\|_{L_{2}\left(\Omega_{c}\right)}^{2}+\gamma\|u-F\|_{H^{2}\left(\Omega_{c}\right)}^{2}, u \in H^{2}\left(\Omega_{c}\right), \\
& \text { subject to the lateral Cauchy data (4.3) })
\end{aligned}
$$

Similarly with Theorems 3.1, 3.2 and using the Carleman estimate of Lemma 4.1, we obtain Theorems 4.1, 4.2. We rely in these theorems on (4.4)-(4.12).

Theorem 4.1. For every $\gamma \in(0,1)$ there exists unique minimizer $u_{\gamma} \in H^{2}\left(\Omega_{c}\right)$ of the functional $J_{\gamma}^{P}(u)$ in (4.11), (4.12) and with a constant $C_{8}=C_{8}\left(\Omega_{c}, P, \rho_{1}\right)>0$ the following estimate holds

$$
\left\|u_{\gamma}\right\|_{H^{2}\left(\Omega_{c}\right)} \leq \frac{C_{8}}{\sqrt{\gamma}}\left(\|F\|_{H^{2}\left(\Omega_{c}\right)}+\|f\|_{L_{2}\left(\Omega_{c}\right)}\right)
$$

Theorem 4.2 (convergence rate). Assume that conditions (2.35) are satisfied. Let in (4.11) the regularization parameter $\gamma=\gamma(\delta)=\delta^{2 \alpha}$, where $\alpha=$ const. $\in(0,1]$. Let $\varepsilon>0$ be a sufficiently small number and the domain $\Omega_{c+3 \varepsilon}$ be as in (4.8). Let the number $m=a^{-\nu_{0}}$, where

$\nu_{0}=\nu_{0}\left(a, c, \mu_{1}, \mu_{2}, \max _{i, j}\left\|a_{i, j}\right\|_{C^{1}\left(\bar{\Omega}_{c}\right)}, X, T\right)>1$ is the number of Lemma 4.1. Define the number $\beta \in(0,1)$ as $\beta=(2 \varepsilon) /(3 m+2 \varepsilon)$. Then there exists a sufficiently small number $\delta_{0}=\delta_{0}\left(\varepsilon, \nu_{0}, P, \Omega_{c}, \rho_{1}\right) \in(0,1)$ and a constant $C_{9}=C_{9}\left(\varepsilon, \nu_{0}, P, \Omega_{c}, \rho_{1}\right)>0$ such that if $\delta \in\left(0, \delta_{0}^{1 / \alpha}\right)$, then the following convergence rate is valid

$$
\left\|u_{\gamma(\delta)}-u^{*}\right\|_{H^{1}\left(\Omega_{c+3 \varepsilon}\right)} \leq C_{9}\left(1+\left\|u^{*}\right\|_{H^{2}\left(\Omega_{c}\right)}\right) \delta^{\alpha \beta}, \forall \delta \in\left(0, \delta_{0}\right),
$$

where $u_{\gamma(\delta)}$ is the minimizer of the functional 4.11), 4.11), which is guaranteed by Theorem 4.1 .

\section{Parabolic Equation With The Reversed Time}

While we had the lateral Cauchy data in section 4 , now we have the data at $\{t=0\}$. Furthermore, instead of the above Hölder stability estimates in subdomains, we obtain below the logarithmic stability estimate for the function $u(x, T)$, which means an estimate in the whole domain. Thus, we need to reformulate the general scheme of section 2 and provide new proofs of analogs of Theorems 2.1-2.5. It should be pointed out that if we would estimate the solution for $t \in(0, T-\varepsilon)$ for a small $\varepsilon>0$, rather than at $\{t=T\}$, then we would have the Hölder stability, see Theorem 1 in $\S 2$ of Chapter 4 of the book Lavrentiev, Romanov and Shishatskii [64] and estimate (5.20) below. However, we follow 
here a modified version of the paper of the author [53] and get the logarithmic stability in the whole domain this way. The Carleman estimate of Lemma 3 of [53] is a modification of the Carleman estimate of Lemma 3 of $\S 2$ of Chapter 4 of [64]. The same is true for Lemma 5.3 below. The key element of this modification, which is absent in [64], is the first term in the third line of (5.10). Indeed, after the integration over $t \in(0, T)$, this term provides a positive integral involving $u^{2}$ over $\{t=T\}$ : because we choose in Lemma $5.3 k+T<a_{0}$, where the number $a_{0}>0$ is sufficiently small. That positive integral, in turn allows us to obtain the logarithmic stability estimate for the problem considered in this section. Still, since [53] is concerned with estimates of initial conditions of parabolic PDEs with lateral Cauchy data, rather then with the parabolic equation with reversed time, we need to modify results of that paper here.

We refer to books of Isakov [36] and Payne [68] for the so-called "logarithmic convexity" method, which provides Hölder stability estimates for solutions of parabolic equations with the reversed time for $u(x, T-\varepsilon)$ in the case when the elliptic operator of that parabolic equation is self adjoint. Also, exercise 3.1.2 of [36] guarantees the logarithmic stability for $u(x, T)$ under the assumption that the norm $\|u(x, t)\|_{L_{2}(\Omega)}$ is uniformly bounder for $t \in[0, T]$. We do not use this assumption here.

\subsection{Problem statement}

Again, let $G \subset \mathbb{R}^{n}$ be a bounded domain with a piecewise smooth boundary and $T=$ const. $>0$. We now denote $Q_{T}=G \times(0, T), S_{T}=\partial G \times(0, T)$. Similarly with section 4, let $L_{\text {par }}$ be the elliptic operator of the second order in $Q_{T}$, whose coefficients depend on $x, t$ and satisfy conditions (3.3), (3.4) where $\Omega$ is replaced with $Q_{T}$ and the dependence on $x$ is replaced with the dependence on $x, t$. Let $L_{0, p a r}$ be the principal part of the operator $L$, like in (3.2). Let the function $f(x, t) \in L_{2}\left(Q_{T}\right)$. Consider the parabolic equation with the reversed time in $Q_{T}$, supplied by an initial condition and a Dirichlet boundary condition,

$$
\begin{aligned}
u_{t}+L_{p a r} u & =f \text { in } Q_{T}, u \in H^{2}\left(Q_{T}\right), \\
u(x, 0) & =g(x), \\
& \left.u\right|_{S_{T}}=p(x, t) .
\end{aligned}
$$

Even though $u \in H^{2,1}\left(Q_{T}\right)$ in (5.1) sounds more natural than $u \in H^{2}\left(Q_{T}\right)$, we still need this extra smoothness in (5.1) for the derivation of the stability estimate of Theorem 5.1 as well as for the convergence rate in Theorem 5.3. In fact, the method presented in this section 5 enables one to replace the Dirichlet boundary condition (5.3) with the Neumann boundary condition. However, we are not doing this here for brevity. In the elliptic operator $L_{\text {par }}$, let $C\left(\bar{Q}_{T}\right)$-norms of coefficients at lower order terms be bounded by a positive constant $M$,

$$
\left\|b_{j}\right\|_{C\left(\bar{Q}_{T}\right)} \leq M, j=0, \ldots, n .
$$

The Parabolic Problem With The Reversed Time. Given conditions (5.1)(5.3), find the function $u(x, T)$.

It is well known that this problem is ill-posed. Indeed, consider, for example the 
following problem

$$
\begin{aligned}
v_{t}+v_{x x} & =0,(x, t) \in(0, \pi) \times(0, T), \\
v(x, 0) & =r(x), \\
v(0, t) & =v(\pi, t)=0 .
\end{aligned}
$$

Let $r_{n}$ be Fourier coefficients of the function $r(x)$ with respect to the functions $\{\sin n x\}_{n=1}^{n}$. Then

$$
v(x, t)=\sum_{n=1}^{\infty} r_{n} \sin n x e^{n^{2} t} .
$$

It follows from (5.5) that small perturbations of the function $r(x)$ can cause large perturbations of the function $v(x, t)$. Also, the solution of this problem exists only for a rather narrow set of functions $r(x)$ : for those, for which the series (5.5) converges. Likewise, the larger $t$ is, the more unstable the solution is. The latter is reflected in the fact that the Carleman estimate of Lemma 5.3 below enables us to use only small values of $T$.

\subsection{The Carleman estimate}

Let $k=$ const. $>0$ be the number which we choose in Lemma 5.3. For $\lambda>1$ we now define the Carleman Weight Function $\varphi_{\lambda}(t)$ as

$$
\varphi_{\lambda}(t)=(k+t)^{-\lambda}, t>0 .
$$

Thus, level hypersurfaces of this function are hyperplanes $\{t=$ const. $\}$. Lemmata 5.1 and 5.2 are reformulations of Lemmata 1 and 2 respectively of [53]. Hence, we do not prove them for brevity.

Lemma 5.1. There exists a sufficiently large number

$$
\lambda_{0}=\lambda_{0}\left(\mu_{1}, \mu_{2}, \max _{i, j}\left\|a_{i, j}\right\|_{C^{1}\left(\bar{\Omega}_{c}\right)}\right)>1 \text { and a constant } C=C\left(\mu_{1}, \mu_{2}, \max _{i, j}\left\|a_{i, j}\right\|_{C^{1}\left(\bar{\Omega}_{c}\right)}\right)>
$$

0 such that for all $\lambda \geq \lambda_{0}$ and for all functions $u \in C^{2,1}\left(\bar{Q}_{T}\right)$ the following estimate holds with the function $\varphi_{\lambda}(t)$ from (5.6) and for all $(x, t) \in Q_{T}$

$$
\begin{aligned}
\left(-u_{t}-L_{0, p a r} u\right) u \varphi_{\lambda}^{2} & \geq \mu_{1}|\nabla u|^{2} \varphi_{\lambda}^{2}-\lambda u^{2} \varphi_{\lambda}^{2}+\operatorname{div} U_{1}+\frac{\partial}{\partial t}\left(-\frac{u^{2}}{2} \varphi_{\lambda}^{2}\right) \\
\left|U_{1}\right| & \leq C|u||\nabla u| \varphi_{\lambda}^{2}
\end{aligned}
$$

Lemma 5.2. For numbers $\lambda_{0}, C$ of Lemma 5.1, for all $\lambda \geq \lambda_{0}$ and for all functions $u \in C^{2,1}\left(\bar{Q}_{T}\right)$ the following estimate holds with the function $\varphi_{\lambda}(t)$ from (5.6) for all $(x, t) \in Q_{T}$

$$
\begin{gathered}
\left(u_{t}+L_{0, p a r} u\right)^{2} \varphi_{\lambda}^{2} \geq-C|\nabla u|^{2} \varphi_{\lambda}^{2}+\lambda(k+t)^{-2} u^{2} \varphi_{\lambda}^{2}+ \\
\frac{\partial}{\partial t}\left(\lambda(k+t)^{-1} u^{2} \varphi_{\lambda}^{2}-\varphi_{\lambda}^{2} \sum_{i, j=1}^{n} a_{i, j} u_{x_{i}} u_{x_{j}}\right)+\operatorname{div} U_{2} \\
\left|U_{2}\right| \leq C\left|u_{t}\right||\nabla u| \varphi_{\lambda}^{2} .
\end{gathered}
$$

To obtain the Carleman estimate out of these two lemmata, we should combine them. This is done in Lemma 5.3. Although the dependence of numbers $\lambda_{1}, \theta, C_{1}$ on the number 
$M$ defined in (5.4) is not necessary in Lemma 5.3, we still include this dependence in its formulation in (??), since we need it in the proof of Theorem 5.1.

Lemma 5.3 (Carleman estimate). There exists a sufficiently small number

$a_{0}=a_{0}\left(\mu_{1}, \mu_{2}, \max _{i, j}\left\|a_{i, j}\right\|_{C^{1}\left(\bar{\Omega}_{c}\right)}, M\right) \in(0,1)$ such that if $k+T<a_{0}$, then there exists a sufficiently large number $\lambda_{1}=\lambda_{1}\left(\mu_{1}, \mu_{2}, \max _{i, j}\left\|a_{i, j}\right\|_{C^{1}\left(\bar{\Omega}_{c}\right)}, M\right)$ and constants $C=C\left(\mu_{1}, \mu_{2}, \max _{i, j}\left\|a_{i, j}\right\|_{C^{1}\left(\bar{\Omega}_{c}\right)}\right)>0, C_{1}=C_{1}\left(\mu_{1}, \mu_{2}, \max _{i, j}\left\|a_{i, j}\right\|_{C^{1}\left(\bar{\Omega}_{c}\right)}\right)>0$ and $\theta=\theta\left(\mu_{1}, \mu_{2}, \max _{i, j}\left\|a_{i, j}\right\|_{C^{1}\left(\bar{\Omega}_{c}\right)}, M\right) \quad$ such that for all $\lambda \geq \lambda_{1}$ and for all functions $u \in C^{2,1}\left(\bar{Q}_{T}\right)$ the following estimate holds with the function $\varphi_{\lambda}(t)$ from (5.6) for all $(x, t) \in Q_{T}$

$$
\begin{gathered}
\left(u_{t}+L_{0, p a r} u\right)^{2} \varphi_{\lambda}^{2} \geq \frac{2}{3} \theta \mu_{1}|\nabla u|^{2} \varphi_{\lambda}^{2}+C \lambda u^{2} \varphi_{\lambda}^{2}+\operatorname{div} U \\
+\frac{\partial}{\partial t}\left(C \lambda(k+t)^{-1}\left(1-C_{1}(k+t)\right) u^{2} \varphi_{\lambda}^{2}-C \varphi_{\lambda}^{2} \sum_{i, j=1}^{n} a_{i, j} u_{x_{i}} u_{x_{j}}\right), \\
|U| \leq C\left(\left|u_{t}\right|+|u|\right)|\nabla u| \varphi_{\lambda}^{2} .
\end{gathered}
$$

Proof. Choose a number $\theta=\theta\left(\mu_{1}, \mu_{2}, \max _{i, j}\left\|a_{i, j}\right\|_{C^{1}\left(\bar{\Omega}_{c}\right)}, M\right)>0$ such that $\theta \mu_{1}>$ $2 C$. Multiply (5.7) by $\theta$ and sum up with (5.10). We obtain

$$
\begin{aligned}
& \left(u_{t}+L_{0, p a r} u\right)^{2} \varphi_{\lambda}^{2}-\theta\left(u_{t}+L_{0, p a r} u\right) u \varphi_{\lambda}^{2} \\
\geq & \frac{\theta \mu_{1}}{2}|\nabla u|^{2} \varphi_{\lambda}^{2}+\lambda(k+t)^{-2}\left(1-\theta(k+t)^{2}\right) u^{2} \varphi_{\lambda}^{2} \\
& +\frac{\partial}{\partial t}\left[\lambda(k+t)^{-1}\left(1-\frac{\theta}{2}(k+t)\right) u^{2} \varphi_{\lambda}^{2}-\varphi_{\lambda}^{2} \sum_{i, j=1}^{n} a_{i, j} u_{x_{i}} u_{x_{j}}\right]+\operatorname{div} U .
\end{aligned}
$$

In (5.11) $U=\theta U_{1}+U_{2}$. Hence, using estimate (5.8) as well as estimate of the third line of (5.9), we obtain the estimate of the third line of (5.10) for $|U(x, t)|$. Choose the number $a_{0}$ so small that

$$
\theta a_{0}^{2}<1 / 2
$$

Since $k+T<a_{0}$, then (5.11) implies that

$$
\begin{aligned}
& \left(u_{t}+L_{0, p a r} u\right)^{2} \varphi_{\lambda}^{2}-\theta\left(u_{t}+L_{0, p a r} u\right) u \varphi_{\lambda}^{2} \\
\geq & \frac{\theta \mu_{1}}{2}|\nabla u|^{2} \varphi_{\lambda}^{2}+\frac{\lambda}{2 a_{0}^{2}} u^{2} \varphi_{\lambda}^{2} \\
& +\frac{\partial}{\partial t}\left[\lambda(k+t)^{-1}\left(1-C_{1}(k+t)\right) u^{2} \varphi_{\lambda}^{2}-\varphi_{\lambda}^{2} \sum_{i, j=1}^{n} a_{i, j} u_{x_{i}} u_{x_{j}}\right]+\operatorname{div} U .
\end{aligned}
$$

Next, by Cauchy-Schwarz inequality

$$
\left(u_{t}+L_{0, p a r} u\right)^{2} \varphi_{\lambda}^{2}-\theta\left(u_{t}+L_{0, p a r} u\right) u \varphi_{\lambda}^{2} \leq \frac{3}{2}\left(u_{t}+L_{0, p a r} u\right)^{2} \varphi_{\lambda}^{2}+\frac{\theta^{2}}{2} u^{2} \varphi_{\lambda}^{2} .
$$

Replacing the left hand side of (5.13) with the right hand side of (5.14), we obtain (5.10). 


\subsection{Stability estimates}

For a sufficiently small parameter $\delta \in(0,1)$ consider the family of functions $u_{\delta}$ satisfying the following conditions

$$
\begin{aligned}
\int_{Q_{T}}\left(\partial_{t} u_{\delta}+L_{p a r} u_{\delta}\right)^{2} d x d t & \leq N \delta^{2}, u_{\delta} \in H^{2}\left(Q_{T}\right), \\
\left\|u_{\delta}(x, 0)\right\|_{L_{2}(\Omega)} & \leq \sqrt{N} \delta \\
u_{\delta} & \mid S_{T}=0
\end{aligned}
$$

where the constant $N>0$ is independent on $\delta$. Conditions (5.15)-(5.17) are generalizations of conditions (5.1) $-(5.3)$ for the case when $p(x, t) \equiv 0$ and $L_{2}$-norms of functions $f, g$ are sufficiently small.

Theorem 5.1 (stability estimates). Consider the family of functions $u_{\delta}$ satisfying conditions (5.15)-(5.17). Let the number $T$ is so small that $2 T<a_{0}$, where the sufficiently small number $a_{0}=a_{0}\left(\mu_{1}, \mu_{2}, \max _{i, j}\left\|a_{i, j}\right\|_{C^{1}\left(\bar{\Omega}_{c}\right)}, M\right) \in(0,1)$ was defined in Lemma 5.3. Then there exists a sufficiently small number $\delta_{0}=\delta_{0}\left(L_{p a r}\right) \in(0,1)$ and a number $C_{10}=C_{10}\left(\mu_{1}, \mu_{2}, \max _{i, j}\left\|a_{i, j}\right\|_{C^{1}\left(\bar{\Omega}_{c}\right)}, M, N, Q_{T}\right)>0$ such that the following logarithmic stability estimate holds for functions $u_{\delta}(x, T)$

$$
\left\|u_{\delta}(x, T)\right\|_{L_{2}(\Omega)} \leq \frac{C_{10}}{\sqrt{\ln \left(\delta^{-1}\right)}}\left(1+\left\|u_{\delta}\right\|_{H^{2}\left(Q_{T}\right)}\right) .
$$

For every $\varepsilon \in(0, T / 2)$ define the number $\beta=\beta(\varepsilon)$ as

$$
\beta=\beta(\varepsilon)=-\frac{\ln (1-\varepsilon / T)}{2 \ln (1-\varepsilon / 2 T)} \in\left(0, \frac{1}{2}\right) .
$$

Then the following Hölder stability estimate holds

$$
\left\|\nabla u_{\delta}\right\|_{L_{2}\left(Q_{T-\varepsilon}\right)}+\left\|u_{\delta}\right\|_{L_{2}\left(Q_{T-\varepsilon}\right)} \leq C_{10}\left(1+\left\|u_{\delta}\right\|_{H^{2}\left(Q_{T}\right)}\right) \delta^{\beta} .
$$

Proof. In this proof $C=C\left(\mu_{1}, \mu_{2}, \max _{i, j}\left\|a_{i, j}\right\|_{C^{1}\left(\bar{\Omega}_{c}\right)}\right)$ denotes different positive constants depending on listed parameters and the number $k=$ const. $\in(0, T]$. By (5.6) $\max _{[0, T]} \varphi_{\lambda}^{2}(t)=k^{-2 \lambda}$. Hence, by (5.15)

$$
\begin{aligned}
N k^{-2 \lambda} \delta^{2} \geq & \int_{Q_{T}}\left(\partial_{t} u_{\delta}+L_{p a r} u_{\delta}\right)^{2} \varphi_{\lambda}^{2} d x d t \geq \\
& \int_{Q_{T}}\left(\partial_{t} u_{\delta}+L_{0, p a r} u_{\delta}\right)^{2} \varphi_{\lambda}^{2} d x d t-C M^{2} \int_{Q_{T}}\left(\left|\nabla u_{\delta}\right|^{2}+u_{\delta}^{2}\right) \varphi_{\lambda}^{2} d x d t .
\end{aligned}
$$

Integrate (5.10) over $Q_{T}$ with the function $u:=u_{\delta}$ in it. Even though in (5.10) $u \in$ $C^{2,1}\left(\bar{Q}_{T}\right)$ while $u_{\delta} \in H^{2}\left(Q_{T}\right)$, this can be handled by density arguments. It follows from the Gauss formula, the third line of (5.10) and (5.17) that the boundary integral over $S_{T}$ is zero in this case. Hence, we obtain

$$
\int_{Q_{T}}\left(\partial_{t} u_{\delta}+L_{0, p a r} u_{\delta}\right)^{2} \varphi_{\lambda}^{2} d x d t \geq \frac{2}{3} \theta \mu_{1} \int_{Q_{T}}\left|\nabla u_{\delta}\right|^{2} \varphi_{\lambda}^{2} d x d t+C_{10} \lambda \int_{Q_{T}} u_{\delta}^{2} \varphi_{\lambda}^{2} d x d t
$$




$$
\begin{aligned}
& +(k+T)^{-2 \lambda} \int_{\Omega}\left(C \lambda(k+T)^{-1}\left(1-C_{1}(k+T)\right) u_{\delta}^{2}-C \sum_{i, j=1}^{n} a_{i, j} \partial_{x_{i}} u_{\delta} \partial_{x_{j}} u\right)(x, T) d x \\
& +k^{-2 \lambda} \int_{\Omega}\left(-C \lambda k\left(1-C_{1} k\right) u_{\delta}^{2}+C \sum_{i, j=1}^{n} a_{i, j} \partial_{x_{i}} u_{\delta} \partial_{x_{j}} u\right)(x, 0) d x .
\end{aligned}
$$

Hence, by (5.21)

$$
\begin{gathered}
N k^{-2 \lambda} \delta^{2} \geq \frac{2}{3} \theta \mu_{1} \int_{Q_{T}}\left|\nabla u_{\delta}\right|^{2} \varphi_{\lambda}^{2} d x d t+C_{10} \lambda \int_{Q_{T}} u_{\delta}^{2} \varphi_{\lambda}^{2} d x d t \\
-C M^{2} \int_{Q_{T}}\left(\left|\nabla u_{\delta}\right|^{2}+u_{\delta}^{2}\right) \varphi_{\lambda}^{2} d x d t \\
+(k+T)^{-2 \lambda} \int_{\Omega}\left(C \lambda(k+T)^{-1}\left(1-C_{1}(k+T)\right) u_{\delta}^{2}-C \sum_{i, j=1}^{n} a_{i, j} \partial_{x_{i}} u_{\delta} \partial_{x_{j}} u\right)(x, T) d x \\
+k^{-2 \lambda} \int_{\Omega}\left(-C \lambda k\left(1-C_{1} k\right) u_{\delta}^{2}+C \sum_{i, j=1}^{n} a_{i, j} \partial_{x_{i}} u_{\delta} \partial_{x_{j}} u\right)(x, 0) d x .
\end{gathered}
$$

Choose $\lambda_{2}=\lambda_{2}\left(L_{p a r}\right) \geq \lambda_{1}$ so large that $C_{10} \lambda>2 C M^{2}$. Next, choose

$\theta=\theta\left(\mu_{1}, \mu_{2}, \max _{i, j}\left\|a_{i, j}\right\|_{C^{1}\left(\bar{\Omega}_{c}\right)}, M\right)$ so large that $\left(\theta \mu_{1}\right) / 3>C M^{2}$. Next, choose $a_{0}>0$ so small that $C_{1} a_{0}<1 / 4$ and also (5.12) would be satisfied. Recalling that $2 T<a_{0}, k \in(0, T)$, using (5.16) ) and (5.22) and taking into account (3.4), we obtain

$$
\begin{gathered}
\int_{Q_{T}}\left(\partial_{t} u_{\delta}+L_{0, p a r} u_{\delta}\right)^{2} \varphi_{\lambda}^{2} d x d t \geq \frac{1}{3} \theta \mu_{1} \int_{Q_{T}}\left|\nabla u_{\delta}\right|^{2} \varphi_{\lambda}^{2} d x d t+C \lambda \int_{Q_{T}} u_{\delta}^{2} \varphi_{\lambda}^{2} d x d t \\
+C \lambda(k+T)^{-2 \lambda-1}\left\|u_{\delta}(x, T)\right\|_{L_{2}(\Omega)}^{2}-C(k+T)^{-2 \lambda}\|\nabla u(x, T)\|_{L_{2}(\Omega)}^{2}-C N \lambda k^{-2 \lambda} \delta^{2} .
\end{gathered}
$$

Hence, (5.21) and (5.23) lead to the following estimate

$$
\begin{aligned}
& C N \lambda k^{-2 \lambda} \delta^{2}+C(k+T)^{-2 \lambda}\|\nabla u(x, T)\|_{L_{2}(\Omega)}^{2} \\
\geq & \frac{1}{3} \theta \mu_{1} \int_{Q_{T}}\left|\nabla u_{\delta}\right|^{2} \varphi_{\lambda}^{2} d x d t+C \lambda \int_{Q_{T}} u_{\delta}^{2} \varphi_{\lambda}^{2} d x d t+C \lambda(k+T)^{-2 \lambda-1}\left\|u_{\delta}(x, T)\right\|_{L_{2}(\Omega)}^{2} .
\end{aligned}
$$

First, we obtain the logarithmic stability estimate (5.18). By the trace theorem, there exists a positive constant $D=D\left(Q_{T}\right)$ such that $\|\nabla w(x, T)\|_{L_{2}(\Omega)}^{2} \leq D\|w\|_{H^{2}\left(Q_{T}\right)}^{2}$. Hence, dividing both sides of (5.24) by $C \lambda(k+T)^{-2 \lambda-1}$, recalling that $k+T \leq 2 T<a_{0}<1$ and ignoring first two terms in the second line of (15.24), we obtain

$$
\left\|u_{\delta}(x, T)\right\|_{L_{2}(\Omega)}^{2} \leq C_{10}\left(1+\frac{T}{k}\right)^{2 \lambda} \delta^{2}+\frac{1}{\lambda} C_{10}\left\|u_{\delta}\right\|_{H^{2}\left(Q_{T}\right)}^{2} .
$$


Set $k=T$ and choose $\lambda=\lambda(\delta)$ such that $2^{2 \lambda}=1 / \delta$. Hence,

$$
\lambda(\delta)=\ln \left(\frac{1}{\delta}\right)^{1 /(2 \ln 2)} .
$$

Naturally, we assume that $\delta$ is so small that $\lambda(\delta) \geq \lambda_{2}$. Hence, (5.25) and (5.26) imply that

$$
\left\|u_{\delta}(x, T)\right\|_{L_{2}(\Omega)}^{2} \leq \frac{C_{10}}{\ln \left(\delta^{-1}\right)}\left(1+\left\|u_{\delta}\right\|_{H^{2}\left(Q_{T}\right)}^{2}\right) .
$$

This, in turn implies (5.18).

We now prove the Hölder stability estimate (5.20). Recall that we have now $k=T$. Since $\varphi_{\lambda}^{2}(t) \geq(2 T-\varepsilon)^{-2 \lambda}$ for $t \in(0, T-\varepsilon)$, then ignoring the last term in the second line of (5.24), we obtain from (5.24) with a different constant $C$

$$
\left\|\nabla u_{\delta}\right\|_{L_{2}\left(Q_{T-\varepsilon}\right)}^{2}+\left\|u_{\delta}\right\|_{L_{2}\left(Q_{T-\varepsilon}\right)}^{2} \leq C_{10}\left(2-\frac{\varepsilon}{T}\right)^{2 \lambda} \delta^{2}+C_{10}\left(1-\frac{\varepsilon}{2 T}\right)^{2 \lambda}\left\|u_{\delta}\right\|_{H^{2}\left(Q_{T}\right)}^{2} .
$$

Since $\varepsilon \in(0, T / 2)$, then $2-\varepsilon / T>1$. Hence, assuming that $\delta$ is sufficiently small, we can choose a different $\lambda=\lambda(\delta)$ such that $(2-\varepsilon / T)^{2 \lambda}=1 / \delta$. Hence,

$$
\lambda(\delta)=\frac{1}{2 \ln (2-\varepsilon / T)} \ln \left(\frac{1}{\delta}\right) .
$$

For every $\varepsilon \in(0, T / 2)$, we have $(1-\varepsilon /(2 T))^{2 \lambda(\delta)}=\delta^{2 \beta}$, where $\beta=\beta(\varepsilon) \in(0,1 / 2)$ is the number defined in (5.19). Hence, (5.27) and (5.28) imply that

$$
\left\|\nabla u_{\delta}\right\|_{L_{2}\left(Q_{T-\varepsilon}\right)}^{2}+\left\|u_{\delta}\right\|_{L_{2}\left(Q_{T-\varepsilon}\right)}^{2} \leq C_{10}\left(1+\left\|u_{\delta}\right\|_{H^{2}\left(Q_{T}\right)}^{2}\right) \delta^{2 \beta} .
$$

Theorem 5.2 follows immediately from Theorem 5.1.

Theorem 5.2 (uniqueness). There exists at most one solution of the problem (5.1)(5.3).

\subsection{Regularization}

We now construct the Tikhonov functional for the problem (5.1)-(5.3). Suppose that there exists a function $F \in H^{2}\left(Q_{T}\right)$ such that

$$
F(x, 0)=g(x),\left.F\right|_{S_{T}}=p(x, t) .
$$

The Tikhonov functional for the problem (5.1)-(5.3) is

$$
\begin{aligned}
J_{\gamma, L_{p a r}}(u)= & \left\|u_{t}+L_{p a r} u\right\|_{L_{2}\left(Q_{T}\right)}^{2}+\gamma\|u-F\|_{H^{2}\left(Q_{T}\right)}^{2}, u \in H^{2}\left(Q_{T}\right), \\
& \text { subject to conditions (15.3) and (15.3). }
\end{aligned}
$$

To establish the convergence rate of minimizers, we again assume the existence of the exact solution $u^{*} \in H^{2}\left(Q_{T}\right)$ of the problem (5.1)-(5.3). This solution satisfies conditions (5.1) -(5.3) with the exact data $g^{*}, p^{*}, f^{*}$. Hence, there exists a function $F^{*} \in H^{2}\left(Q_{T}\right)$ satisfying conditions (5.29) in which $g$ and $p$ are replaced with $g^{*}$ and $p^{*}$ respectively. We assume that

$$
\left\|g-g^{*}\right\|_{L_{2}(G)} \leq \delta,\left\|f-f^{*}\right\|_{L_{2}\left(Q_{T}\right)},\left\|F-F^{*}\right\|_{H^{2}\left(Q_{T}\right)} \leq \delta
$$


Theorem 5.3 follows immediately from Theorem 2.4. Theorem 5.4 follows immediately from Theorem 2.5, Remark 2.1 and Theorem 5.1.

Theorem 5.3 (existence). Suppose that there exists a function $F \in H^{2}\left(Q_{T}\right)$ satisfying conditions (5.29). Then for each $\gamma>0$ there exists unique minimizer $u_{\gamma} \in H^{2}\left(Q_{T}\right)$ of the functional (5.30), (5.31). Furthermore, with a constant $C_{11}=C_{11}\left(L_{p a r}, Q_{T}\right)$ the following estimate holds

$$
\left\|u_{\gamma}\right\|_{H^{2}\left(Q_{T}\right)} \leq \frac{C_{11}}{\sqrt{\gamma}}\left(\|F\|_{H^{2,1}\left(Q_{T}\right)}+\|f\|_{L_{2}\left(Q_{T}\right)}\right)
$$

Theorem 5.4 (convergence rate). Assume that conditions (5.32) hold. Let in (5.30) the regularization parameter $\gamma=\gamma(\delta)=\delta^{2 \alpha}$, where $\alpha=$ const. $\in(0,1]$. Let the number $T$ is so small that $T<a_{0} / 2$, where the sufficiently small number

$a_{0}=a_{0}\left(\mu_{1}, \mu_{2}, \max _{i, j}\left\|a_{i, j}\right\|_{C^{1}\left(\bar{\Omega}_{c}\right)}, M\right) \in(0,1)$ was defined in Lemma 5.3. Let the number $\varepsilon \in(0, T / 2)$ and let $\beta=\beta(\varepsilon) \in(0,1 / 2)$ be the number defined in (5.19). Then there exists a sufficiently small number $\delta_{0}=\delta_{0}\left(L_{\text {par }}, a_{0}, Q_{T}\right) \in(0,1)$ and a constant $C_{12}=C_{12}\left(L_{\text {par }}, a_{0}, Q_{T}\right)>0$ such that for all $\delta \in\left(0, \delta_{0}^{1 / \alpha}\right)$ the following convergence rates are valid

$$
\begin{gathered}
\left\|u_{\gamma(\delta)}(x, T)-u^{*}(x, T)\right\|_{L_{2}(\Omega)} \leq \frac{C_{12}}{\sqrt{\ln \left(\delta^{-1}\right)}}\left(1+\left\|u^{*}\right\|_{H^{2}\left(Q_{T}\right)}\right), \\
\left\|\nabla u_{\gamma(\delta)}-\nabla u^{*}\right\|_{L_{2}\left(Q_{T-\varepsilon}\right)}+\left\|u_{\gamma(\delta)}-u^{*}\right\|_{L_{2}\left(Q_{T-\varepsilon}\right)} \leq C_{12}\left(1+\left\|u_{\delta}\right\|_{H^{2}\left(Q_{T}\right)}\right) \delta^{\alpha \beta},
\end{gathered}
$$

where $u_{\gamma(\delta)}$ is the minimizer of the functional (5.30), (5.31), which is guaranteed by Theorem 5.3.

\section{Hyperbolic Equation With Lateral Cauchy Data}

Results of this section were originate in the work of Klibanov and Malinsky [48] and were developed further in works of Klibanov with coauthors [29, 46, 49, 52, 54, 56]. As in subsection 5.1 , let $G \subset \mathbb{R}^{n}$ be a bounded domain with a piecewise smooth boundary $\partial G$ and $T=$ const. $>0$. Denote

$$
Q_{T}=G \times(0, T), S_{T}=\partial G \times(0, T), Q_{T}^{ \pm}=G \times(-T, T), S_{T}^{ \pm}=\partial G \times(-T, T) .
$$

In this section we obtain both the Lipschitz type stability (Theorem 6.1) and the Lipschitz type convergence rate (Theorem 6.3) in the whole time cylinder $Q_{T}^{ \pm}$rather than weaker Hölder type estimates in subdomains, as in previous sections. As it was mentioned in Introduction, corresponding numerical studies of [29, 49, 54] have demonstrated a good performance.

The Carleman estimate of Lemma 6.1 for the hyperbolic operator $L_{0, \text { hyp }}=a(x) \partial_{t}^{2}-\Delta$ was proved in Theorem 1.10.2 of [9]. Other forms of Carleman estimates for the hyperbolic case can be found in, e.g. Theorem 3.4.1 of [36], Theorem 2.2.4 of [52], Lemma 2 of $\S 4$ of chapter 4 of [64] and in Lemma 3.1 [72]. 


\subsection{Problem statement}

Let numbers $a_{l}, a_{u}>0$ and $a_{l}<a_{u}$. For $x \in G$, let the function $a(x)$ satisfy the following conditions in $G$

$$
a(x) \in\left[a_{l}, a_{u}\right], a \in C^{1}(\bar{G}) .
$$

In addition, we assume that there exists a point $x_{0} \in G$ such that

$$
\left(\nabla a(x), x-x_{0}\right) \geq \alpha=\text { const. }>0, \forall x \in \bar{G},
$$

where $(\cdot, \cdot)$ denotes the scalar product in $\mathbb{R}^{n}$. We need inequality (6.3) for the validity of the Carleman estimate of Lemma 6.1. Also, let functions

$$
b_{j}(x, t) \in C\left(\bar{Q}_{T}^{ \pm}\right), j=0, \ldots, n ; M=\max _{j}\left\|b_{j}\right\|_{C\left(\bar{Q}_{T}^{ \pm}\right)} .
$$

Let the function $f \in L_{2}\left(Q_{T}^{ \pm}\right)$and the function $u$ satisfies the following conditions

$$
\begin{aligned}
& L_{h y p} u=a(x) u_{t t}-\Delta u-\sum_{j=1}^{n} b_{j}(x, t) u_{x_{j}}-b_{0}(x, t) u=f(x, t) \text { in } Q_{T}^{ \pm}, u \in H^{2}\left(Q_{\text {责 } \oint .5)}\right. \\
& u \quad\left|S_{T}^{ \pm}=g_{0}(x, t), \partial_{n} u\right|_{S_{T}^{ \pm}}=g_{1}(x, t) .
\end{aligned}
$$

Cauchy Problem with the Lateral Data for the Hyperbolic Equation (6.5)). Let coefficients of the hyperbolic operator $L_{h y p}$ satisfy conditions (6.2)-(6.4). Find the function $u$ satisfying conditions (6.5), (6.6) $)$.

Let the number $\eta \in(0,1)$. Let $\lambda>1$ be a large parameter. Define functions

$\xi(x, t), \varphi_{\lambda}(x, t)$ as

$$
\xi(x, t)=\left|x-x_{0}\right|^{2}-\eta t^{2}, \varphi_{\lambda}(x, t)=\exp [\lambda \xi(x, t)] .
$$

Following (2.1), for a number $c>0$ define the hypersurface $\xi_{c}$ and the domain $\Omega_{c}$ as

$$
\xi_{c}=\left\{(x, t) \in Q_{T}^{ \pm}: \xi(x, t)=c,\right\}, \Omega_{c}=\left\{(x, t) \in Q_{T}^{ \pm}: \xi(x, t)>c\right\} .
$$

Lemma 6.1 (Carleman estimate). Let $n \geq 2$ and conditions (6.2) be satisfied. Also, assume that there exists a point $x_{0} \in G$ such that (6.3) holds. Let $M$ be the number in 6.4). Denote $P=P\left(x_{0}, G\right)=\max _{x \in \bar{G}}\left|x-x_{0}\right|$. Then there exists a number $\eta_{0}=\eta_{0}\left(G, P, a_{l}, a_{u},\|\nabla a\|_{C(\bar{G})}\right) \in(0,1)$ such that for any $\eta \in\left(0, \eta_{0}\right)$ one can choose a sufficiently large number $\lambda_{0}=\lambda_{0}\left(G, P, a_{l}, a_{u},\|\nabla a\|_{C(\bar{G})}, M, \eta_{0}, c\right)>1$ and the number $C_{13}=C_{13}\left(G, P, a_{l}, a_{u},\|\nabla a\|_{C(\bar{G})}, M, \eta_{0}, c\right)>0$, such that for all $u \in C^{2}\left(\bar{\Omega}_{c}\right)$ and for all $\lambda \geq \lambda_{0}$ the following pointwise Carleman estimate holds

$$
\begin{aligned}
\left(L_{\text {hyp }} u\right)^{2} \varphi^{2} & \geq C_{13} \lambda\left(|\nabla u|^{2}+u_{t}^{2}\right) \varphi_{\lambda}^{2}+C_{13} \lambda^{3} u^{2} \varphi_{\lambda}^{2}+\operatorname{div} U+V_{t}, \text { in } \Omega_{c}, \\
|U| & \leq C_{13} \lambda^{3}\left(|\nabla u|^{2}+u_{t}^{2}+u^{2}\right) \varphi_{\lambda}^{2} \\
|V| & \leq C_{13} \lambda^{3}\left[|t|\left(u_{t}^{2}+|\nabla u|^{2}+u^{2}\right)+(|\nabla u|+|u|)\left|u_{t}\right|\right] \varphi_{\lambda}^{2} .
\end{aligned}
$$

In particular, (6.11) implies that if either $u(x, 0)=0$ or $u_{t}(x, 0)=0$, then

$$
V(x, 0)=0 .
$$

In the case $a(x) \equiv 1$ one can choose any $\eta \in(0,1)$ and condition (6.3) is not required. 


\subsection{Lipschitz stability estimate}

We now obtain the Lipschitz stability estimate for a problem, which is more general than the problem (6.5), (6.6). Let the function $u \in H^{2}\left(Q_{T}\right)$ satisfies conditions (6.6) and the following inequality

$$
\int_{Q_{T}^{ \pm}}\left(L_{\text {hyp }} u\right)^{2} d x d t \leq K^{2}
$$

Theorem 6.1. Let conditions (6.3)- (6.4) be satisfied. Suppose that $T>P / \sqrt{\eta_{0}}$, where $\eta_{0}=\eta_{0}\left(G, P, a_{l}, a_{u},\|\nabla a\|_{C(\bar{G})}\right) \in(0,1)$ is the number of Lemma 6.1. Then there exists a constant $C_{14}=C_{14}\left(P, a_{l}, a_{u},\|\nabla a\|_{C(\bar{G})}, M, \eta_{0}, Q_{T}^{ \pm}\right)>0$ such that for any function $u \in H^{2}\left(Q_{T}^{ \pm}\right)$satisfying conditions (6.6), (6.13) the following Lipschitz stability estimate holds

$$
\|u\|_{H^{1}\left(Q_{T}^{ \pm}\right)} \leq C_{14}\left(\left\|g_{0}\right\|_{H^{1}\left(S_{T}^{ \pm}\right)}+\left\|g_{1}\right\|_{L_{2}\left(S_{T}^{ \pm}\right)}+K\right) .
$$

In the case $a(x) \equiv 1$ and one can choose $\eta_{0}=1$, and if in this case $G=\{|x|<R\}$, then one can choose $T>R$.

Proof. In this proof $C_{14}=C_{14}\left(P, a_{l}, a_{u},\|\nabla a\|_{C(\bar{G})}, M, \eta_{0}, Q_{T}^{ \pm}\right)>0$ denotes different positive constants depending on listed parameters. Choose the number $\eta \in\left(0, \eta_{0}\right)$ so close to $\eta_{0}$ that $T>P / \sqrt{\eta}$. Then (6.8) implies that

$$
\Omega_{c} \subset Q_{T}^{ \pm}, \bar{\Omega}_{c} \cap\{t= \pm T\}=\varnothing .
$$

Let $m=\max _{\bar{\Omega}_{c}} \xi(x, t)$. Then $m=\max _{x \in \bar{\Omega}_{c}}\left|x-x_{0}\right|^{2}$. We have

$$
\int_{Q_{T}^{ \pm}}\left(L_{h y p} u\right)^{2} d x d t=\int_{Q_{T}^{ \pm}}\left(L_{h y p} u\right)^{2} \varphi_{\lambda}^{2} \varphi_{\lambda}^{-2} d x d t \geq e^{-2 \lambda m} \int_{Q_{T}^{ \pm}}\left(L_{h y p} u\right)^{2} \varphi_{\lambda}^{2} d x d t .
$$

Hence, using (6.13), we obtain

$$
\int_{Q_{T}^{ \pm}}\left(L_{h y p} u\right)^{2} \varphi_{\lambda}^{2} d x d t \leq K^{2} e^{2 \lambda m}
$$

By (??) and (6.16)

$$
K^{2} e^{2 \lambda m} \geq \int_{Q_{T}^{ \pm}}\left(L_{h y p} u\right)^{2} \varphi_{\lambda}^{2} d x d t \geq \int_{Q_{T}^{ \pm}}\left(L_{0, h y p} u\right)^{2} \varphi_{\lambda}^{2} d x d t-C_{14} \int_{Q_{T}^{ \pm}}\left(|\nabla u|^{2}+u^{2}\right)^{2} \varphi_{\lambda}^{2} d x d t .
$$

Hence,

$$
\int_{Q_{T}^{ \pm}}\left(L_{0, h y p} u\right)^{2} \varphi_{\lambda}^{2} d x d t \leq C_{14} \int_{Q_{T}^{ \pm}}\left(|\nabla u|^{2}+u^{2}\right)^{2} \varphi_{\lambda}^{2} d x d t+K^{2} e^{2 \lambda m} .
$$

Let $\omega(x, t)$ be a function such that

$$
\omega \in C^{2}\left(\bar{Q}_{T}^{ \pm}\right), \omega(x, t)=\left\{\begin{array}{c}
1,(x, t) \in \Omega_{c+2 \varepsilon} \\
0,(x, t) \in Q_{T}^{ \pm} \backslash \Omega_{c+\varepsilon} \\
\in[0,1],(x, t) \in \Omega_{c+\varepsilon} \backslash \Omega_{c+2 \varepsilon}
\end{array}\right.
$$


Consider the function $v=\omega u$. Then

$$
L_{0, \text { hyp }} v=\omega L_{0, \text { hyp }} u+2\left(a(x) \omega_{t} u_{t}-\nabla \omega \nabla u\right)+u L_{0, \text { hyp }} \omega .
$$

Hence, using (6.17), we obtain

$$
\int_{Q_{T}^{ \pm}}\left(L_{0, h y p} v\right)^{2} \varphi_{\lambda}^{2} d x d t \leq C_{14} \int_{Q_{T}^{ \pm}}\left(|\nabla u|^{2}+u^{2}\right)^{2} \varphi_{\lambda}^{2} d x d t+K^{2} e^{2 \lambda m} .
$$

On the other hand, integrate (6.9) over $\Omega_{c}$ for the function $v$, using (6.10), (6.11), (6.15), Gauss formula and the fact that by (6.8) and (6.18) $\left.v\right|_{\xi_{c}}=0$. We obtain

$$
\begin{aligned}
\int_{Q_{T}^{ \pm}}\left(L_{0, h y p} v\right)^{2} \varphi_{\lambda}^{2} d x d t \geq & \int_{\Omega_{c}}\left(L_{0, h y p} v\right)^{2} \varphi_{\lambda}^{2} d x d t \geq C_{14} \int_{\Omega_{c}}\left(\lambda\left(|\nabla v|^{2}+v_{t}^{2}\right)+\lambda^{3} v^{2}\right) \varphi_{\lambda}^{2} d x d t \\
& -C_{14} \lambda^{3} \int_{\partial \Omega_{c} \cap S_{T}^{ \pm}}\left(|\nabla v|^{2}+v_{t}^{2}+v^{2}\right) \varphi_{\lambda}^{2} d S .
\end{aligned}
$$

Next, by (6.6)

$$
-C_{14} \lambda^{3} \int_{\partial \Omega_{c} \cap S_{T}^{ \pm}}\left(|\nabla v|^{2}+v_{t}^{2}+v^{2}\right) \varphi_{\lambda}^{2} d S \geq-C_{14} \lambda^{3} e^{2 \lambda m}\left(\left\|g_{0}\right\|_{H^{1}\left(Q_{T}^{ \pm}\right)}^{2}+\left\|g_{1}\right\|_{L_{2}\left(Q_{T}^{ \pm}\right)}^{2}\right) .
$$

Also, since $\Omega_{c+2 \varepsilon} \subset \Omega_{c}$ and by (6.18), $v=u$ in $\Omega_{c+2 \varepsilon}$ and then

$$
C_{14} \int_{\Omega_{c}}\left(\lambda\left(|\nabla v|^{2}+v_{t}^{2}\right)+\lambda^{3} v^{2}\right) \varphi_{\lambda}^{2} d x d t \geq C_{14} \lambda \int_{\Omega_{c+2 \varepsilon}}\left(|\nabla u|^{2}+u^{2}\right) \varphi_{\lambda}^{2} d x d t
$$

Comparing this inequality with (6.19), (6.20) and (6.21), we obtain

$$
\begin{aligned}
\lambda \int_{\Omega_{c+2 \varepsilon}}\left(|\nabla u|^{2}+u_{t}^{2}+u^{2}\right) \varphi_{\lambda}^{2} d x d t \leq & C_{14} \int_{Q_{T}^{ \pm}}\left(|\nabla u|^{2}+u^{2}\right)^{2} \varphi_{\lambda}^{2} d x d t \\
& +C_{14}\left(\left\|g_{0}\right\|_{H^{1}\left(Q_{T}^{ \pm}\right)}^{2}+\left\|g_{1}\right\|_{L_{2}\left(Q_{T}^{ \pm}\right)}^{2}+K^{2}\right) e^{3 \lambda m}
\end{aligned}
$$

Next,

$$
\begin{gathered}
C_{14} \int_{Q_{T}^{ \pm}}\left(|\nabla u|^{2}+u_{t}^{2}+u^{2}\right)^{2} \varphi_{\lambda}^{2} d x d t= \\
C_{14} \int_{\Omega_{c+2 \varepsilon}}\left(|\nabla u|^{2}+u_{t}^{2}+u^{2}\right)^{2} \varphi_{\lambda}^{2} d x d t+C_{14} \int_{Q_{T}^{ \pm} \backslash \Omega_{c+2 \varepsilon}}\left(|\nabla u|^{2}+u_{t}^{2}+u^{2}\right)^{2} \varphi_{\lambda}^{2} d x d t \\
\leq C_{14} \int_{\Omega_{c+2 \varepsilon}}\left(|\nabla u|^{2}+u_{t}^{2}+u^{2}\right)^{2} \varphi_{\lambda}^{2} d x d t+C_{14} e^{2 \lambda(c+2 \varepsilon)} \int_{Q_{T}^{ \pm} \backslash \Omega_{c+2 \varepsilon}}\left(|\nabla u|^{2}+u_{t}^{2}+u^{2}\right)^{2} d x d t .
\end{gathered}
$$


Comparing this with (6.22) and taking $\lambda \geq \max \left(\lambda_{0}, \lambda_{1}\right)$, where $\lambda_{1}=C_{14} / 2$, we obtain

$$
\begin{aligned}
& \lambda \int_{\Omega_{c+2 \varepsilon}}\left(|\nabla u|^{2}+u_{t}^{2}+u^{2}\right) \varphi_{\lambda}^{2} d x d t \leq C_{14} e^{2 \lambda(c+2 \varepsilon)} \int_{Q_{T}^{ \pm} \backslash \Omega_{c+2 \varepsilon}}\left(|\nabla u|^{2}+u_{t}^{2}+u^{2}\right)^{2} d x(6 t 23) \\
& +C_{14}\left(\left\|g_{0}\right\|_{H^{1}\left(Q_{T}^{ \pm}\right)}^{2}+\left\|g_{1}\right\|_{L_{2}\left(Q_{T}^{ \pm}\right)}^{2}+K^{2}\right) e^{3 \lambda m} .
\end{aligned}
$$

We have $\Omega_{c+3 \varepsilon} \subset \Omega_{c+2 \varepsilon}$ and $\varphi_{\lambda}^{2} \geq \exp (2 \lambda(c+3 \varepsilon))$ in $\Omega_{c+3 \varepsilon}$. Hence,

$$
\lambda \int_{\Omega_{c+2 \varepsilon}}\left(|\nabla u|^{2}+u_{t}^{2}+u^{2}\right) \varphi_{\lambda}^{2} d x d t \geq \lambda e^{2 \lambda(c+3 \varepsilon)} \int_{\Omega_{c+3 \varepsilon}}\left(|\nabla u|^{2}+u_{t}^{2}+u^{2}\right) d x d t .
$$

Substituting this inequality in (6.23) and dividing by $\lambda e^{2 \lambda(c+3 \varepsilon)}$, we obtain

$$
\|u\|_{H^{1}\left(\Omega_{c+3 \varepsilon}\right)}^{2} \leq C_{14} e^{-2 \lambda \varepsilon}\|u\|_{H^{1}\left(Q_{T}^{ \pm}\right)}^{2}+C_{14}\left(\left\|g_{0}\right\|_{H^{1}\left(S_{T}^{ \pm}\right)}^{2}+\left\|g_{1}\right\|_{L_{2}\left(S_{T}^{ \pm}\right)}^{2}+K^{2}\right) e^{3 \lambda m} .
$$

Or

$$
\|u\|_{H^{1}\left(\Omega_{c+3 \varepsilon}\right)} \leq C_{14} e^{-\lambda \varepsilon}\|u\|_{H^{1}\left(Q_{T}^{ \pm}\right)}+C_{14}\left(\left\|g_{0}\right\|_{H^{1}\left(S_{T}^{ \pm}\right)}+\left\|g_{1}\right\|_{L_{2}\left(S_{T}^{ \pm}\right)}+K\right) e^{(3 \lambda m) / 2} .
$$

We now temporary indicate the dependence of the domain $\Omega_{c}$ on the point $x_{0}$, i.e. $\Omega_{c}\left(x_{0}\right)$. There exists a sufficiently small number $c=c\left(x_{0},\|a\|_{C^{1}(\bar{G})}\right)>0$ and a sufficiently small number $\varepsilon>0$ such that

$$
\begin{aligned}
\left\{\left|x-x_{0}\right| \leq 2 \sqrt{c+3 \varepsilon}\right\} & \subset G, \\
\left(\nabla a(x), x-x_{0}^{\prime}\right) & \geq \frac{\alpha}{2}>0, \forall x \in \bar{G}, \forall x_{0}^{\prime} \in\left\{\left|x_{0}-x_{0}^{\prime}\right| \leq 2 \sqrt{c+3 \varepsilon}\right\},
\end{aligned}
$$

see (6.3). Choose a point $x_{0}^{\prime}$ such that $\left|x_{0}-x_{0}^{\prime}\right|=2 \sqrt{c+3 \varepsilon}$. Consider a point $x \in G$ such that $\left|x-x_{0}\right|<\sqrt{c+3 \varepsilon}$. Hence, the point $(x, 0) \notin \bar{\Omega}_{c+3 \varepsilon}\left(x_{0}\right)$. On the other hand, by the triangle inequality

$$
\left|x-x_{0}^{\prime}\right|=\left|x-x_{0}-\left(x_{0}^{\prime}-x_{0}\right)\right| \geq\left|x_{0}-x_{0}^{\prime}\right|-\left|x-x_{0}\right|>2 \sqrt{c+3 \varepsilon}-\sqrt{c+3 \varepsilon}=\sqrt{c+3 \varepsilon} .
$$

Hence, $(x, 0) \in \Omega_{c+3 \varepsilon}\left(x_{0}^{\prime}\right)$. This means that $G \subset\left(\Omega_{c+3 \varepsilon}\left(x_{0}\right) \cup \Omega_{c+3 \varepsilon}\left(x_{0}^{\prime}\right)\right)$. Hence, there exists a sufficiently small number $\sigma>0$ such that $Q_{\sigma}^{ \pm} \subset\left(\Omega_{c+3 \varepsilon}\left(x_{0}\right) \cup \Omega_{c+3 \varepsilon}\left(x_{0}^{\prime}\right)\right):=Y$. Clearly

$$
\|u\|_{H^{1}\left(Q_{\sigma}^{ \pm}\right)} \leq\|u\|_{H^{1}(Y)} \leq\|u\|_{H^{1}\left(\Omega_{c+3 \varepsilon}\left(x_{0}\right)\right)}+\|u\|_{H^{1}\left(\Omega_{c+3 \varepsilon}\left(x_{0}^{\prime}\right)\right)} .
$$

Hence, using in the left hand side of (6.24) $\|u\|_{H^{1}\left(\Omega_{c+3 \varepsilon}\left(x_{0}\right)\right)}$ first and $\|u\|_{H^{1}\left(\Omega_{c+3 \varepsilon}\left(x_{0}^{\prime}\right)\right)}$ and adding those two inequalities, we obtain

$$
\|u\|_{H^{1}\left(Q_{\sigma}^{ \pm}\right)} \leq C_{14} e^{-\lambda \varepsilon}\|u\|_{H^{1}\left(Q_{T}^{ \pm}\right)}+C_{14}\left(\left\|g_{0}\right\|_{H^{1}\left(S_{T}^{ \pm}\right)}+\left\|g_{1}\right\|_{L_{2}\left(S_{T}^{ \pm}\right)}+K\right) e^{(3 \lambda m) / 2} .
$$

Hence, there exists a number $t_{0} \in(-\sigma, \sigma)$ such that

$$
\int_{\Omega}\left(|\nabla u|^{2}+u_{t}^{2}+u^{2}\right)\left(x, t_{0}\right) d x \leq
$$




$$
C_{14}\left[e^{-2 \lambda \varepsilon}\|u\|_{H^{1}\left(Q_{T}^{ \pm}\right)}^{2}+\left(\left\|g_{0}\right\|_{H^{1}\left(Q_{T}^{ \pm}\right)}^{2}+\left\|g_{1}\right\|_{L_{2}\left(Q_{T}^{ \pm}\right)}^{2}+K^{2}\right) e^{3 \lambda m}\right] .
$$

Let $y(x, t)=L_{\text {hyp }} u$. Then $y \in L_{2}\left(Q_{T}^{ \pm}\right)$and

$$
\begin{aligned}
L_{h y p} u & =y(x, t) \text { in } Q_{T}^{ \pm}, \\
u\left(x, t_{0}\right)= & u_{0}(x), u_{t}\left(x, t_{0}\right)=u_{1}(x), \\
u & \left|S_{T}^{ \pm}=g_{0}(x, t), \partial_{n} u\right|_{S_{T}^{ \pm}}=g_{1}(x, t) .
\end{aligned}
$$

We now refer to the classical method of energy estimates, see, e.g. chapter 4 in the book of Ladyzhenskaya [60]. First, consider conditions (6.26)-(6.28) as the initial boundary value problem in $Q_{t_{0}, T}=\Omega \times\left(t_{0}, T\right)$. Then the method of energy estimates gives

$$
\begin{aligned}
& \|u\|_{H^{1}\left(Q_{t_{0}, T}\right)}^{2} \\
\leq & C_{14}\left(\left\|u_{0}\right\|_{H^{1}(\Omega)}^{2}+\left\|u_{1}\right\|_{L_{2}(\Omega)}^{2}+\left\|g_{0}\right\|_{H^{1}\left(\partial \Omega \times\left(t_{0}, T\right)\right)}^{2}+\left\|g_{1}\right\|_{H^{1}\left(\partial \Omega \times\left(t_{0}, T\right)\right)}^{2}+\|y\|_{L_{2}\left(Q_{t_{0}, T}\right)}^{2}\right) .
\end{aligned}
$$

Next, since time can be reversed in hyperbolic PDEs, we consider conditions (6.26) $-(6.28)$ as the initial boundary value problem in $Q_{t_{0},-T}=\Omega \times\left(-T, t_{0}\right)$. Then again the energy estimate leads to

$$
\begin{aligned}
& \|u\|_{H^{1}\left(Q_{t_{0},-T}\right)}^{2} \\
\leq & C_{14}\left(\left\|u_{0}\right\|_{H^{1}(\Omega)}^{2}+\left\|u_{1}\right\|_{L_{2}(\Omega)}^{2}+\left\|g_{0}\right\|_{H^{1}\left(\partial \Omega \times\left(-T, t_{0}\right)\right)}^{2}+\left\|g_{1}\right\|_{H^{1}\left(\partial \Omega \times\left(-T, t_{0}\right)\right)}^{2}+\|y\|_{L_{2}\left(Q_{t_{0},-T}\right)}^{2}\right) .
\end{aligned}
$$

Summing up the last two inequalities, we obtain

$$
\begin{aligned}
& \|u\|_{H^{1}\left(Q_{T}^{ \pm}\right)}^{2} \\
\leq & C_{14}\left(\left\|u_{0}\right\|_{H^{1}(\Omega)}^{2}+\left\|u_{1}\right\|_{L_{2}(\Omega)}^{2}+\left\|g_{0}\right\|_{H^{1}\left(S_{T}^{ \pm}\right)}^{2}+\left\|g_{1}\right\|_{H^{1}\left(S_{T}^{ \pm}\right)}^{2}+\|y\|_{L_{2}\left(Q_{T}^{ \pm}\right)}^{2}\right) .
\end{aligned}
$$

By (6.13) $\|y\|_{L_{2}\left(Q_{T}^{ \pm}\right)}^{2} \leq K^{2}$. Hence, (6.25) and (6.29) lead to

$$
\|u\|_{H^{1}\left(Q_{T}^{ \pm}\right)}^{2} \leq C_{14}\left[e^{-2 \lambda \varepsilon}\|u\|_{H^{1}\left(Q_{T}^{ \pm}\right)}^{2}+\left(\left\|g_{0}\right\|_{H^{1}\left(Q_{T}^{ \pm}\right)}^{2}+\left\|g_{1}\right\|_{L_{2}\left(Q_{T}^{ \pm}\right)}^{2}+K^{2}\right) e^{3 \lambda m}\right] .
$$

Choose $\lambda_{2} \geq \max \left(\lambda_{0}, \lambda_{1}\right)$ such that $C_{14} e^{-2 \lambda_{2} \varepsilon}<1 / 2$. Then (6.30) implies that

$$
\|u\|_{H^{1}\left(Q_{T}^{ \pm}\right)}^{2} \leq C_{14}\left(\left\|g_{0}\right\|_{H^{1}\left(Q_{T}^{ \pm}\right)}^{2}+\left\|g_{1}\right\|_{L_{2}\left(Q_{T}^{ \pm}\right)}^{2}+K^{2}\right) e^{3 \lambda_{2} m}
$$

This immediately leads to the target estimate (6.14) with a new constant $C_{14}$.

Consider now the case $a(x) \equiv 1, G=\{|x|<R\}$. Since by Lemma 6.1 one can take $\alpha=0$ in (6.3) in this case, then we choose $x_{0}=0$ and then follow the above proof.

\subsection{Regularization}

Recall that we need to find an approximate solution $u$ of the problem (6.5), (6.6)). Just as above, we assume that there exists a function $F$ satisfying the following conditions

$$
F \in H^{2}\left(Q_{T}^{ \pm}\right),\left.F\right|_{S_{T}^{ \pm}}=g_{0}(x, t),\left.\partial_{n} F\right|_{S_{T}^{ \pm}}=g_{1}(x, t) .
$$


Let (, ) and [,] be scalar products in $L_{2}\left(Q_{T}^{ \pm}\right)$and $H^{2}\left(Q_{T}^{ \pm}\right)$respectively and let $\|\cdot\|$ and $[\cdot]$ be respective norms. We find an approximate solution of the problem (6.5), (6.6) via the minimization of the following Tikhonov functional

$$
\begin{aligned}
J_{\gamma}(u)= & \left\|L_{\text {hyp }} u-f\right\|^{2}+\gamma[u-F]^{2}, \\
& \text { subject to the lateral Cauchy data (6.6). }
\end{aligned}
$$

Theorem 6.2 (uniqueness and existence of the minimizer). Assume that there exists a function $F$ satisfying conditions (6.31). Then for every $\gamma \in(0,1)$ there exists unique minimizer $u_{\gamma} \in H^{2}\left(Q_{T}^{ \pm}\right)$of the functional (6.32), (6.33) and the following estimate holds

$$
\left[u_{\gamma}\right] \leq \frac{C_{14}}{\sqrt{\gamma}}\left([F]+\|f\|_{L_{2}\left(Q_{T}^{ \pm}\right)}\right)
$$

where $C_{14}=C_{14}\left(P, a_{l}, a_{u},\|\nabla a\|_{C(\bar{G})}, M, \eta, Q_{T}^{ \pm}\right)>0$ is the constant of Theorem 6.1.

The proof of this theorem is similar with the proof of Theorem 2.4 and is, therefore, omitted. Again, to estimate the convergence rate of minimizers, we assume that there exists the exact solution $u^{*} \in H^{2}\left(Q_{T}^{ \pm}\right)$of the problem (6.5), (6.6) with the exact right hand side $f^{*}$ in (6.5) and exact lateral Cauchy data $g_{0}^{*}, g_{1}^{*}$ in (6.6). Hence, there exists a function $F^{*}$ satisfying conditions (6.31) with $g_{0}^{*}, g_{1}^{*}$.

Theorem 6.3 (convergence rate). For $\gamma \in(0,1)$, let $u_{\gamma} \in H^{2}\left(Q_{T}^{ \pm}\right)$be the unique minimizer of the functional (6.32), (6.33), which is guaranteed by Theorem 6.2. Suppose that there exists a point $x_{0} \in G$ such that condition (6.3) is satisfied. Let the number $P=P\left(x_{0}, G\right)=\max _{x \in \bar{G}}\left|x-x_{0}\right|$. Suppose that $T>P / \sqrt{\eta_{0}}$, where $\eta_{0}=$ $\eta_{0}\left(G, P, a_{l}, a_{u},\|\nabla a\|_{C(\bar{G})}\right) \in(0,1)$ is the number of Lemma 6.1. Then with the constant $C_{14}=C_{14}\left(P, a_{l}, a_{u},\|\nabla a\|_{C(\bar{G})}, M, \eta_{0}, Q_{T}^{ \pm}\right)>0$ of Theorem 6.1 the following estimate holds

$$
\begin{gathered}
\left\|u_{\gamma}-u^{*}\right\|_{H^{1}\left(Q_{T}^{ \pm}\right)} \leq \\
C_{14}\left(\left\|g_{0}-g_{0}^{*}\right\|_{H^{1}\left(S_{T}^{ \pm}\right)}+\left\|g_{1}-g_{1}^{*}\right\|_{L_{2}\left(S_{T}^{ \pm}\right)}+\left\|f-f^{*}\right\|+\left[F-F^{*}\right]+\sqrt{\gamma}\left[u^{*}\right]\right),
\end{gathered}
$$

where $u_{\gamma}$ is the minimizer of the functional (6.32), (6.33), which is guaranteed by Theorem 6.2. In particular, let $\delta \in(0,1), \gamma=\gamma(\delta)=\delta^{2}$ and let

$$
\left\|g_{0}-g_{0}^{*}\right\|_{H^{1}\left(S_{T}^{ \pm}\right)},\left\|g_{1}-g_{1}^{*}\right\|_{L_{2}\left(S_{T}^{ \pm}\right)},\left\|f-f^{*}\right\|,\left[F-F^{*}\right] \leq \delta .
$$

Then (6.34) becomes

$$
\left\|u_{\gamma(\delta)}-u^{*}\right\|_{H^{1}\left(Q_{T}^{ \pm}\right)} \leq C_{14}\left(1+\left[u^{*}\right]\right) \delta .
$$

In the case $a(x) \equiv 1$ and $G=\{|x|<R\}$ condition (6.3) is not required and estimates 6.34), (6.35) are valid for $T>R$.

Proof. We need to prove this theorem, since, unlike all above convergence results, we have $\delta^{1}$ in (6.35) instead of $\delta^{\varkappa}$ with a certain $\varkappa \in(0,1)$. Thus, the convergence rate is stronger here than in above theorems. Denote $\widetilde{u}_{\gamma}=u^{*}-u_{\gamma}, \widetilde{f}=f^{*}-f, \widetilde{F}=F^{*}-F$. Then we obtain similarly with (2.39)

$$
\begin{aligned}
\left\|L_{h y p} \widetilde{u}_{\gamma}\right\|^{2}+\gamma\left[\widetilde{u}_{\gamma}\right]^{2} \leq & \frac{1}{2}\left\|L_{h y p} \widetilde{u}_{\gamma}\right\|^{2}+\frac{1}{2}\left\|\widetilde{f}-L_{h y p} \widetilde{F}\right\|^{2} \\
& +\frac{\gamma}{2}\left[u^{*}\right]^{2}+\frac{\gamma}{2}\left[\widetilde{u}_{\gamma}\right]^{2} .
\end{aligned}
$$


Hence,

$$
\left\|L_{\text {hyp }} \widetilde{u}_{\gamma}\right\|^{2} \leq\left\|\tilde{f}-L_{\text {hyp }} \widetilde{F}\right\|^{2}+\gamma\left[u^{*}\right]^{2},
$$

Estimates (6.34), (6.35) follow immediately from (6.37) and Theorem 6.1. The statement about the removal of the multiplier $1 / \sqrt{\gamma}$ follows from (6.34). The special case $a(x) \equiv 1$, $G=\{|x|<R\}$ follows from (6.37) and Theorem 6.1.

\section{Thermoacoustic tomography}

In this section we show how results of section 6 can be applied to the problem of the reconstruction of one of initial conditions of a hyperbolic PDE from boundary measurements. This problem is called nowadays "the problem of thermoacoustic tomography". Although results of this section actually follow from the earlier work of Klibanov and Malinsky [48] (1991), this problem did not have that name at that time. More details can be found in the paper of the author [55]. Numerical studies by the method of this section were performed in [29, 54].

\subsection{Statement of the inverse problem}

We assume below that the domain $G$ is a ball of the radius $R, G=\left\{x \in \mathbb{R}^{n}:|x|<R\right\}$ and we keep notations (6.1). Although a more general domain can be considered along the same lines, we are not doing so for brevity. Denote $D_{T}^{n+1}=\mathbb{R}^{n} \times(0, T)$. Let the function $u \in H^{2}\left(D_{T}^{n+1}\right)$ be the solution of the following Cauchy problem

$$
\begin{gathered}
\widehat{L}_{h y p}(u)=a(x) u_{t t}-\Delta u-\sum_{j=1}^{n} b_{j}(x) u_{x_{j}}-b_{0}(x) u=0 \text { in } D_{T}^{n+1}, \\
u(x, 0)=f(x), u(x, 0)=0 .
\end{gathered}
$$

We impose the following conditions on coefficients of equation (7.1)

$$
\begin{aligned}
a & \in C^{1}\left(\mathbb{R}^{n}\right), a(x)=1 \text { and for } x \in \mathbb{R}^{n} \backslash G, \\
b_{j} & \in C\left(\mathbb{R}^{n}\right) \text { and } b_{j}(x)=0, j=0, \ldots, n \text { for } x \in \mathbb{R}^{n} \backslash G, \\
B & =\max _{j}\left\|b_{j}\right\|_{C(\bar{G})} .
\end{aligned}
$$

In addition, we impose conditions (6.2) on the coefficient $a(x)$. Finally, we assume that

$$
f \in H^{3}\left(\mathbb{R}^{n}\right) \text { and } f(x)=0 \text { for } x \in \mathbb{R}^{n} \backslash G .
$$

Corollary 4.1 of $\S 4$ of Chapter 4 of the book of Ladyzhenskaya [60] guarantees that there exists unique solution $u \in H^{3}\left(D_{T}^{n+1}\right)$ of the Cauchy problem (7.1), (17.2), as long as conditions (7.3), (7.6) are satisfied.

Inverse Problem 1. Find the initial condition $f(x)$ assuming that the function $p(x, t)$ is given, where

$$
\left.u\right|_{S_{T}}=p(x, t) .
$$

Hence, $p \in H^{2}\left(S_{T}\right)$ in the case of exact data. In the case of real measurements, the function $p$ is given with a noise. However, it can be smoothed out by a number 
of well known procedures, so that the resulting function belongs to $H^{2}\left(S_{T}\right)$. This is an inverse problem of finding the initial condition from boundary measurements. In the case $b_{j}(x) \equiv 0, j=0, \ldots, n$ this problem is called sometimes "the problem of thermoacoustic tomography". Following the technique of section 6 , we need to figure out the Neumann boundary condition at $S_{T}$ and to estimate it somehow via the function $p(x, t)$. Instead, we will consider a ball, which is both concentric with $G$ and larger than $G$, find both Dirichlet and Neumann boundary conditions on its boundary and estimate them through $p(x, t)$.

Let $\sigma>0$ be a number. Denote

$$
\begin{gathered}
G^{\sigma}=\left\{x \in \mathbb{R}^{n}:|x|<R+\sigma\right\}, Q_{T}^{\sigma}=G^{\sigma} \times(0, T), S_{T}^{\sigma}=\partial G^{\sigma} \times(0, T), \\
g_{0}^{\sigma}(x, t)=\left.u\right|_{S_{T}^{\sigma}}, g_{1}^{\sigma}(x, t)=\left.\partial_{n} u\right|_{S_{T}^{\sigma}} .
\end{gathered}
$$

Similarly with the above, below in this section $C_{15}=C_{15}\left(a_{l}, a_{u}, B, R, \sigma, T\right)>0$ denotes different positive constants depending on listed parameters.

Lemma 7.1. Let conditions (6.2), (7.1)-(7.8) be satisfied. Also, let the function $p \in H^{2}\left(S_{T}\right)$. Then there exists a number $\bar{\sigma} \in(0, \sigma)$ and a number

$C_{15}=C_{15}\left(a_{l}, a_{u}, B, R, \sigma, T\right)>0$ such that

$$
\left\|g_{0}^{\bar{\sigma}}\right\|_{H^{1}\left(S_{T}^{\bar{\sigma}}\right)} \leq C_{15}\|p\|_{H^{2}\left(S_{T}\right)},\left\|g_{1}^{\bar{\sigma}}\right\|_{L_{2}\left(S_{T}^{\bar{\sigma}}\right)} \leq C_{15}\|p\|_{H^{2}\left(S_{T}\right)} .
$$

Proof. For $r>0$, consider the function $\phi_{1}(r)$ such that

$$
\phi_{1}(r) \in C^{2}[0, \infty), \phi_{1}(r)=\left\{\begin{array}{c}
1, r \in[0, R+2 \sigma], \\
\in(0,1), r \in(R+2 \sigma, R+3 \sigma), \\
0, r \geq R+3 \sigma .
\end{array}\right.
$$

For $|x| \geq R$ consider functions $q(x, t)$ and $v(x, t)$ defined as

$$
q(x, t)=\phi_{1}(|x|) p(x, t), v(x, t)=u(x, t)-q(x, t) .
$$

Then (7.1)-(7.7) and (7.10) imply that the function is the solution of the following initial boundary value problem outside of the ball $G$

$$
\begin{aligned}
\widehat{L}_{h y p}(v) & =-\widehat{L}_{h y p}(q),(x, t) \in\left(\mathbb{R}^{n} \backslash G\right) \times(0, T), \\
v(x, 0) & =v_{t}(x, 0)=0, x \in \mathbb{R}^{n} \backslash G, \\
v & \mid S_{T}=0 .
\end{aligned}
$$

Also, $v \in H^{2}\left(\left(\mathbb{R}^{n} \backslash G\right) \times(0, T)\right)$. Consider a ball $G^{\prime}=\left\{x \in \mathbb{R}^{n}:|x|<R^{\prime}\right\}$ where the number $R^{\prime}=R^{\prime}\left(a_{l}, a_{u}, B, \sigma, R, T\right)>R+3 \sigma$ is so large that $u(x, t)=0$ for $(x, t) \in$ $\left\{|x|>R^{\prime} / 2\right\} \times(0, T)$. Such a number $R^{\prime}$ exists due to the finite speed of propagation of solutions of hyperbolic equations, see $\S 2$ of chapter 4 of the book of Ladyzhenskaya [60]. Applying the method of energy estimates [60] to the problem (7.11) in the domain $P_{T}=$ $\left\{(x, t): R<|x|<R^{\prime}, t \in(0, T)\right\}$, taking into account zero Dirichlet boundary conditions for the function $v$ at $\{|x|=R\} \cup\left\{|x|=R^{\prime}\right\}$ and then taking into account (7.10), we obtain

$$
\|u\|_{H^{1}\left(\left(G^{\sigma} \backslash G\right) \times(0, T)\right)} \leq\|u\|_{H^{1}\left(P_{T}\right)} \leq C_{15}\|p\|_{H^{2}\left(S_{T}\right)} .
$$

Since there exists a number $\bar{\sigma} \in(0, \sigma)$ such that

$$
\|u\|_{H^{1}\left(S_{T}^{\bar{\sigma}}\right)},\left\|\partial_{n} u\right\|_{L_{2}\left(S_{T}^{\bar{\sigma}}\right)} \leq \frac{1}{\sigma}\|u\|_{H^{1}\left(\left(G^{\sigma} \backslash G\right) \times(0, T)\right)},
$$

then (17.12) completes the proof. 


\subsection{Lipschitz stability}

Theorem 7.1. Let $\sigma>0$ be a number. Let conditions (6.2), (6.3), (7.1)-(7.7) be satisfied. Let the function $u \in H^{3}\left(D_{T}^{n+1}\right)$ be the solution of the problem (7.1), (7.2) and let (7.7) be valid. Let the number $P=P\left(x_{0}, R\right)=\max _{x \in \bar{G}}\left|x-x_{0}\right|$. Then there exists a number $\eta_{0}=\eta_{0}\left(R, x_{0}, a_{l}, a_{u},\|\nabla a\|_{C(\bar{G})}\right) \in(0,1)$ such that if $T>P / \sqrt{\eta_{0}}$, then there exists a number $C_{15}=C_{15}\left(a_{l}, a_{u}, B, R, \sigma, \eta_{0}, T\right)>0$ such that the following Lipschitz stability estimate holds $\|f\|_{L_{2}(G)} \leq C_{15}\|p\|_{H^{2}\left(S_{T}\right)}$.

Proof. Let $\bar{\sigma} \in(0, \sigma)$ be the number of Lemma 7.1. To apply Theorem 6.1, we replace in it first $G$ with $G^{\bar{\sigma}}$. We notice that when we integrate the pointwise Carleman estimate (6.9) in the proof of Theorem 6.1 over the domain $\Omega_{c} \cap\{t>0\}$ and use the Gauss' formula, the boundary integral over $\Omega_{c} \cap\{t=0\}$ equals zero because of (6.12) and also because $u_{t}(x, 0)=0$ by (7.2). The rest of the proof is identical to the rest of the proof of Theorem 6.1. Hence, using Lemma 7.1, we obtain $\|u\|_{H^{1}\left(G^{\bar{\sigma}} \times(0, T)\right)} \leq C_{15}\|p\|_{H^{2}\left(S_{T}\right)}$. Since $G \subset G^{\bar{\sigma}}$, then by the trace theorem $\|f\|_{L_{2}(G)} \leq C_{15}\|p\|_{H^{2}\left(S_{T}\right)}$.

The fact that this theorem depends on a number $\sigma>0$ is a minor issue in this context. The author believes that this dependence can be eliminated. Since this likely would require an extensive effort, the author is not doing this here.

\subsection{Regularization}

We would need to use now $G^{\bar{\sigma}}$. However, for brevity we replace here $G^{\bar{\sigma}}$ with $G$. This also makes sense from the computational point of view [29, 54]. Indeed, in order to solve Inverse Problem 1 in practical computations, one should first solve the initial boundary value problem in $\left(\mathbb{R}^{n} \backslash G\right) \times(0, T)$,

$$
\begin{aligned}
\widehat{L}_{h y p}(u)= & 0,(x, t) \in\left(\mathbb{R}^{n} \backslash G\right) \times(0, T), \\
u(x, 0)= & u_{t}(x, 0)=0, x \in \mathbb{R}^{n} \backslash G, \\
u & \mid S_{T}=p(x, t) .
\end{aligned}
$$

This way one finds the function $\bar{p}(x, t)=\left.\partial_{n} u\right|_{S_{T}}$. Hence, we construct now a numerical method for Inverse Problem 2. Let

$$
\begin{aligned}
\widehat{L}_{h y p}(u)= & 0 \text { in } Q_{T}, u \in H^{2}\left(Q_{T}\right), \\
u & \left|\quad S_{T}=p(x, t), \partial_{n} u\right|_{S_{T}}=\bar{p}(x, t), \\
u_{t}(x, 0)= & 0 .
\end{aligned}
$$

Inverse Problem 2. Suppose that functions $p, \bar{p}$ in (17.14) are given. Determine the function $f(x)=u(x, 0)$ for $x \in G$ from conditions (7.13)-(7.15).

The difference between the problem (7.13)-(7.15) and the problem (6.5), (6.6) is that now we require one initial condition (7.15). We replace conditions (7.3) $-(7.5)$ with

$$
a \in C^{1}(\bar{G}), b_{j} \in C(\bar{G}), B=\max _{j}\left\|b_{j}\right\|_{C(\bar{G})}, j=0, \ldots, n .
$$

Suppose that there exists a function $F \in H^{2}\left(Q_{T}\right)$ such that

$$
\left.F\right|_{S_{T}}=p(x, t),\left.\partial_{n} F\right|_{S_{T}}=\bar{p}(x, t), F_{t}(x, 0)=0 .
$$


Similarly with (6.32), (6.33) introduce the following Tikhonov functional with the regularization parameter $\gamma \in(0,1)$

$$
\begin{aligned}
\bar{J}_{\gamma}(u)= & \left\|\widehat{L}_{h y p}(u)\right\|_{L_{2}\left(Q_{T}\right)}^{2}+\gamma\|u-F\|_{H^{2}\left(Q_{T}\right)}^{2}, \\
& \text { subject to conditions (17.14) },(15.15) .
\end{aligned}
$$

Theorem 7.2 is a full analog of Theorem 2.4. Therefore, we omit its proof.

Theorem 7.2 (uniqueness and existence of the minimizer). Assume that there exists a function $F$ satisfying conditions (7.17). Then for every $\gamma \in(0,1)$ there exists unique minimizer $u_{\gamma} \in H^{2}\left(Q_{T}\right)$ of the functional (7.18), (7.19) and the following estimate holds with the constant $C_{15}>0$ depending on the same parameters as in Theorem 6.4

$$
\left\|u_{\gamma}\right\|_{H^{2}\left(Q_{T}\right)} \leq \frac{C_{15}}{\sqrt{\gamma}}\|F\|_{H^{2}\left(Q_{T}\right)} .
$$

To prove convergence of minimizers of the functional (7.18), (7.19), we again introduce the exact solution $u^{*} \in H^{2}\left(Q_{T}\right)$ of the problem (7.13)-(7.15) with exact boundary data $p^{*}, \bar{p}^{*}$. Let $f^{*}(x)=u^{*}(x, 0)$. Then there exists a function $F^{*} \in H^{2}\left(Q_{T}\right)$ satisfying conditions (7.17) with $p^{*}, \bar{p}^{*}$ in them. We omit the proof of Theorem 7.3, since it follows immediately from Theorems 7.1 and 7.2 in the same way as Theorem 6.3 follows from Theorems 6.1 and 6.2 .

Theorem 7.3 (convergence rate). Let conditions (6.2), (7.16) be satisfied and assume the existence of such am point $x_{0} \in G$ that condition (6.3) is satisfied as well. For $\gamma \in$ $(0,1)$, let $u_{\gamma} \in H^{2}\left(Q_{T}\right)$ be the unique minimizer of the functional (7.18), (7.19), which is guaranteed by Theorem 7.2. Denote $P=P\left(x_{0}, G\right)=\max _{x \in \bar{G}}\left|x-x_{0}\right|$. Suppose that $T>P / \sqrt{\eta_{0}}$, where $\eta_{0}=\eta_{0}\left(G, P, a_{l}, a_{u},\|\nabla a\|_{C(\bar{G})}\right) \in(0,1)$ is the number of Lemma 6.1. Then with the constant $C_{16}=C_{16}\left(G, P, a_{l}, a_{u},\|\nabla a\|_{C(\bar{G})}, B, \eta_{0}, T\right)>0$ the following estimates hold

$$
\begin{gathered}
\left\|u_{\gamma}(x, 0)-f^{*}(x)\right\|_{L_{2}(G)},\left\|u_{\gamma}-u^{*}\right\|_{H^{1}\left(Q_{T}\right)} \leq \\
C_{16}\left(\left\|p-p^{*}\right\|_{H^{1}\left(S_{T}\right)}+\left\|\bar{p}-\bar{p}^{*}\right\|_{L_{2}\left(S_{T}\right)}+\left\|F-F^{*}\right\|_{H^{2}\left(Q_{T}\right)}+\sqrt{\gamma}\left\|u^{*}\right\|_{H^{2}\left(Q_{T}\right)}\right),
\end{gathered}
$$

where $u_{\gamma}$ is the minimizer of the functional (7.17), (7.18), which is guaranteed by Theorem 7.2. In particular, let $\delta \in(0,1), \gamma=\gamma(\delta)=\delta^{2}$ and let

$$
\left\|p-p^{*}\right\|_{H^{1}\left(S_{T}\right)},\left\|\bar{p}-\bar{p}^{*}\right\|_{L_{2}\left(S_{T}\right)},\left\|F-F^{*}\right\|_{H^{2}\left(Q_{T}\right)} \leq \delta .
$$

Then (7.20) becomes

$$
\left\|u_{\gamma}(x, 0)-f^{*}(x)\right\|_{L_{2}(G)},\left\|u_{\gamma}-u^{*}\right\|_{H^{1}\left(Q_{T}\right)} \leq C_{16}\left(1+\left\|u^{*}\right\|_{H^{2}\left(Q_{T}\right)}\right) \delta .
$$

In the case $a(x) \equiv 1$ condition (6.3) is not necessary and one can choose $\eta_{0}=1$ and $T>R$. 


\section{Published Results}

In this section we overview main published results about the topic of the current paper: Tikhonov functionals for ill-posed Cauchy problems for PDEs, which are generated by differential operators of those PDEs, under the condition that the corresponding PDO admits a Carleman estimate. We consider both linear and nonlinear problems. In addition to works on this topic of the author with coauthors cited in Introduction, a number of quite elegant results were obtained by Bourgeois and Dardé. They have done this for the Cauchy problem for the Laplace equation and related problems. This effort was initiated by Bourgeois in 2005 [15]. Papers [15, 17, 19, 20, 21, 30] of these authors contain quite good results of numerical experiments. These results are obtained using the FEM. Regular $C^{0}$ finite elements were used in [15, 23, 30]. In [17, 19, 20, 21, 22] non-conforming finite elements were used. Papers [15, 16, 17, 18, 20, 21, 22, 30] work with the variational formulation of the Tikhonov functional for the Cauchy problem for the Laplace equation. That functional is generated by the Laplace operator $\Delta$. The paper [19] works with the variational formulation of the Tikhonov functional generated by the operator $P=\Delta+k, k=$ const. $\in \mathbb{R}$ for the Cauchy problem for the equation $P u=0$.

\subsection{Linear problems}

All above theorems rely on $H^{2}$ spaces. It was observed in [15] that these spaces would lead either to $C^{1}$ finite elements or to finite differences. However, for rather complicated domains finite elements are better applicable than finite differences. On the other hand, since $C^{0}$ finite elements are the most popular ones. Thus, the main idea of the paper [15] is to present a mixed formulation of the QRM, which would enable one to work with standard $C^{0}$ finite elements. Two regularization parameters were used in [15].

It is clear from sections 2-7 that a stability estimate for an ill-posed Cauchy problem implies a similar estimate for the convergence rate of minimizers of the Tikhonov functional generated by the corresponding PDO. In theorems of sections 2-4 rates of convergence of minimizers of Tikhonov functionals are given only in certain subdomains of original domains, and these rates are of the Hölder type. On the other hand, Theorem 5.4 provides the Hölder type convergence rate for a subdomain $Q_{T-\varepsilon}$ of the domain $Q_{T}$ and the logarithmic type convergence rate for the entire domain $Q_{T}$. Thus, one can anticipate that only the logarithmic type convergence rate can be obtained in elliptic and parabolic cases if considering the whole domain. The logarithmic convergence rate in the whole domain was obtained in [18] for the case of the Cauchy problem for the Laplace equation. To get that convergence rate, a result of Phung [69] was used. The result of [69], in turn is based on Carleman estimates. While the result of [69] is valid for domains with the $C^{\infty}$ boundary, in [18] it was generalized for the case of domains of the class $C^{1,1}$. The result of [18] was extended by Bourgeois and Dardé in [19] to the case of Lipschitz domains and for the above operator $P=\Delta+k$.

As to the issue of the $H^{2}$ smoothness of solutions, Bourgeois and Dardé have made a point in [20] that noisy data are not smooth. Thus, they have addressed in [20] the problem of working with non-smooth noisy data by QRM for the case of the Cauchy problem for the Laplace equation. They have used a duality-based approach. Dardé, Hannukainen and Hyvönen [30] have further extended the idea of [15], for the case of the Cauchy problem for an elliptic PDE, in order to work with the standard $C^{0}$ finite 
elements. Furthermore, they have proven, for the first time, a quite intriguing result about the monotonic convergence of regularized solutions of the QRM. In other words, they have proven that a certain norm of a certain difference between the regularized solution and the exact solution strictly monotonically decreases when the regularization parameter $\gamma$ decreases. Another salient feature of [30] is that the first 3-d computations of QRM are presented there, whereas only 2-d cases were considered numerically prior to [30]. Computations in [20, 30], were performed for both cases: the "pure" Cauchy problem and the inverse obstacle problem (see subsection 7.2 for the latter).

Klibanov [53] has studied the problem of determining the initial condition of a general parabolic PDE of the second order from lateral Cauchy data. This is of course the problem in the whole time cylinder rather than in its part. Thus, a logarithmic stability estimate should be anticipated. This estimate was obtained in [53] using Carleman estimates. In this case two Carleman estimates were combined: one for the lateral Cauchy data and the second one for the case of reversed time. In other words, analogs of Lemmata 4.1 and 5.3 respectively were combined. Next, the Tikhonov functional generated by that parabolic operator was constructed and the logarithmic convergence rate of minimizers was established.

\subsection{Nonlinear problems I: Inverse obstacle problems}

Bourgeois and Dardé were the first ones who have applied the QRM to the inverse obstacle problems. Note that these problems are nonlinear, unlike all problems considered above. In a quite elegant work [21] they have proposed a new iterative procedure of the predictorcorrector type. On the predictor step they solve the Cauchy problem for the Laplace equation in a 2-d domain, which is located between the boundary of the original 'large' domain and the boundary of a first guess for the unknown obstacle. On the corrector step they use that QRM solution to update the boundary of the unknown obstacle via a version of the level set method. In [22] and [23] they have extended the idea of [21] to the much more complicated cases of identification of defects for the elastic-plastic constitutive law and the inverse obstacle problem for the Stokes system respectively. Extending the idea of [15], two mixed formulations were used in [23] for the QRM in order to work with the standard Lagrange finite elements.

\subsection{Nonlinear problems II: Coefficient Inverse Problems (CIPs)}

A CIP is about the reconstruction of a coefficient of a PDE from boundary measurements. Both the most important and the most challenging question in a numerical treatment of a CIP is: Is it possible to have a rigorous guarantee of obtaining at least one point in a small neighborhood of the exact solution, provided that this neighborhood is unknown in advance? The author calls a numerical method addressing this question globally convergent. There are currently three types of globally convergent numerical methods which are not only developed analytically but tested numerically as well. First two types work for the case of a single measurement event and the third type works for the case of multiple measurements. First two types of methods are rooted to the original idea of [24], since both eliminate the unknown coefficient from the original PDE via the differentiation with respect to a certain parameter from which this coefficient does not depend. Finally, the third type of globally convergent numerical methods is the method of Kabanikhin and Shishlenin 
[38, 40, 41], which is based on a multidimensional analog of the Gel'fand-Levitan-Krein equation.

The reason of the importance of the topic of global convergence is that conventional least squares functionals for CIPs suffer from the phenomenon of multiple local minima and ravines. Therefore, any optimization technique for such a functional is a locally convergent method, such as, e.g. gradient and Newton methods. In other words, it has a rigorous guarantee of convergence only if its starting point is located in a sufficiently small neighborhood of the correct solution. Section 5.8.4 of [9], as well as publications [57, 66] contain numerical examples showing that locally convergent methods do not converge to the correct solutions even if starting point from the background medium, whereas the globally convergent methods converge. Those examples are for experimental data in [9, 66] and for computationally simulated data in [57].

The first type of globally convergent numerical techniques is the Beilina-Klibanov method for CIPs for the following hyperbolic equation

$$
c(x) u_{t t}=\Delta u, u(x, 0)=0, x \in \mathbb{R}^{3}, t>0 .
$$

This method has been developed since the work [8]. The root in [24] is due to the fact that the unknown coefficient $c(x)$ is "eliminated" from the equation obtained by the Laplace transform of (8.1) via the differentiation with respect to the parameter $s>0$ of this transform and obtaining a nonlinear integral differential equation this way. Global convergence results can be found in [9, 10]. In addition to the convergence theory, this method is currently completely verified on experimental data, see, e.g. [9, 11, 12, 59, 70,

One of procedures of this method is the iterative solution of the boundary value problems for certain elliptic PDEs. Boundary conditions for these problems are generated by the boundary data for the CIP. However, in the case of backscattering data, measurements of the function $u$ are performed only on the backscattering side $\Gamma$ of the boundary $\partial \Omega$ of the domain of interest $\Omega$. This means that those boundary conditions are known only on $\Gamma$ in this case. One way to handle this is a heuristic one: we complement the Dirichlet boundary condition on $\Gamma$ by such a Dirichlet boundary condition on $\partial \Omega \backslash \Gamma$ which is taken from the solution of the forward problem (8.1) for $c(x) \equiv 1$ : we assume that the coefficient $c(x)=1$ for $x \in \mathbb{R}^{3} \backslash \Omega$ and $c(x)$ is unknown in $\Omega$. This way has proved to work well both for backscattering synthetic data [10] and for backscattering experimental data [11, 12, 70]. Furthermore, Chow and Zou [28] have shown numerically on synthetic data that the correct boundary condition on $\partial \Omega \backslash \Gamma$ results in basically the same image as the complemented one as above.

The second way to work with backscattering data by the method of [9] is to solve Cauchy problems for those elliptic equations with Dirichlet and Neumann boundary conditions on $\Gamma$. To solve such a problem, the Tikhonov functional, which is generated by the corresponding elliptic operator, should be minimized, as in section 3. This was done in chapter 6 of [9] for synthetic data. Furthermore, this way has proved to be especially effective for such experimental data, where only one experimental curve per each target was measured, which led to a 1-d CIP [59]. Global convergence theorems for this case were proved in [9, 59] using Carleman estimates. Since the theory was not the main goal of [59], the proof of the global convergence was incomplete in this work. That proof was later completed by Ozbilge [67].

The second type of globally convergent numerical methods was initiated by the author for a CIP for equation (8.1) [50] as well as for the similar parabolic equation [51]. Next, 
this idea was modified by the author and Timonov [52]. Recently there is a renewed interest in this topic for the case of CIPs for equation (8.1), see Beilina and Klibanov [13] and Klibanov and Thành [57].

Loosely speaking, the first step of works [13, 50, 51, 57] is the same as in the method of [24, 26, 45, 56]: the unknown coefficient $c(x)$ is "eliminated" from the original PDE via the differentiation either with respect to $t$, if working in the time domain as in [13, 50, 51], or with respect to the parameter $s$ of the Laplace transform, if working in the "Laplace transform domain" [57]. This way a nonlinear integral differential equation is obtained with respect to a function $w$, which is associated with the function $u(x, t)$ in (8.1). Since both Dirichlet and Neumann boundary conditions are available for $w$, then this can be considered as an ill-posed nonlinear Cauchy problem. The second step is that to solve this problem, a weighted Tikhonov functional $J_{\lambda}(w)$ is constructed. Similarly with the topic of this paper, this functional is generated by the nonlinear integral differential operator of that equation for $w$. The key element of $J_{\lambda}(w)$ is the presence of the Carleman Weight Function (CWF) with the large parameter $\lambda>>1$. The CWF is the one which is involved in the Carleman estimate for a certain associated PDO. The main result then claims that, given a finite set $\Phi(d)$ of an arbitrary diameter $d$ in a certain Hilbert space, one can choose such a value $\lambda_{0}(d)>>1$ that for all $\lambda \geq \lambda_{0}(d)$ the functional $J_{\lambda}(w)$ is strictly convex on the set $\Phi(d)$. In accordance with the Tikhonov concept for ill-posed problems [9, 71], the set $\Phi(d)$ can be considered as the set of admissible parameters and is usually known a priori. The strict convexity of $J_{\lambda}(w)$, in turn implies that the gradient method of the minimization of $J_{\lambda}(w)$ converges to the unique minimizer of $J_{\lambda}(w)$ on the set $\Phi(d)$ if starting from an arbitrary point of this set [13, 57]. Since restrictions on the diameter $d$ of the set $\Phi(d)$ are not imposed, then this is the global convergence. Numerical reconstruction results for computationally simulated data in the 1d case obtained in [57] indicate that this type of methods is promising.

Recently Baudouin, de Buhan and Ervedoza [7] have proposed a similar idea for a CIP for the hyperbolic equation $u_{t t}=\Delta u+q(x) u, x \in \Omega \subset \mathbb{R}^{n}, t>0$ with the unknown potential $q(x)$ and with the data generated by a single measurement event. Here $\Omega$ is a bounded domain. In [7] a sequence of weighted Tikhonov functionals generated by a sequence of linear hyperbolic operators is constructed. As weights, CWFs for the operator $\partial_{t}^{2}-\Delta$ are used. Similarly with the above, it is assumed that the upper bound for the function $q$ is known a priori, i.e. the number $B$ is known, where $\|q\|_{L_{\infty}(\Omega)} \leq B$. As a result, the global convergence to the correct solution $q_{\text {correct }}$ of some functions associated with minimizers of these functionals is proven in [7].

\section{Acknowledgments}

The author is grateful to Professors Anatoly B. Bakushinsky and Laurent Bourgeois for their critical remarks, which have helped to improve the quality of the presentation in this paper. The author is also grateful to Professor Anatoly G. Yagola for prompting him to work on this survey.

\section{References}

[1] S. Andrieux, T. N. Baranger and A. Ben Abda, Solving Cauchy problems by minimizing an energy-like functional, Inverse Problems, 20, 115-133, 2006. 
[2] S. Avdonin, V. Kozlov, D. Maxwell and M. Truffer, Iterative methods for solving a nonlinear boundary inverse problem in glaciology, J. Inverse Ill-Posed Problems, 17, 239-258, 2009.

[3] A.B. Bakushinsky, Difference schemes for the solution of ill-posed abstract Cauchy problems, Differential Equations, 7, 1876-1885, 1971.

[4] A.B. Bakushinsky and A.V. Goncharsky, Ill-posed Problems: Theory and Applications, Kluwer Academic Publ. Boston, 1994

[5] A.B. Bakushinsky, M.Yu. Kokurin and M.M. Kokurin, On a class of finite difference methods for ill-posed Cauchy problems with noisy data, J. Inverse and Ill-Posed Problems, 18, 959-977, 2011.

[6] A.B. Bakushinsky, M.M. Kokurin and M.Yu. Kokurin, On a class of finite difference schemes for solving ill-posed Cauchy problems in Banah spaces, Computational Mathematics and Mathematical Physics, 52, 411-426, 2012.

[7] L. Baudouin, M. de Buhan and S. Ervedoza, Global Carleman estimates for waves and applications, Communications in Partial Differential Equations, 38, 823-859, 2013.

[8] L. Beilina and M.V. Klibanov, A globally convergent numerical method for a coefficient inverse problem, SIAM J. Sci. Comp., 31, 478-509, 2008.

[9] L. Beilina and M.V. Klibanov, Approximate Global Convergence and Adaptivity for Coefficient Inverse Problems, Springer, New York, 2012.

[10] L. Beilina and M.V. Klibanov, A new approximate mathematical model for global convergence for a coefficient inverse problem with backscattering data, J. Inverse and Ill-Posed Problems, 20, 513-565, 2012.

[11] L. Beilina, N. T. Thành, M. V. Klibanov and M. A. Fiddy, Reconstruction from blind experimental data for an inverse problem for a hyperbolic equation, Inverse Problems, 30, 025002, 2014.

[12] L. Beilina, N.T. Thánh, M.V. Klibanov and J.B. Malmberg, Reconstruction of shapes and refractive indices from backscattering experimental data using the adaptivity, Inverse Problems, 30, 105007, 2014.

[13] L. Beilina and M.V. Klibanov, Globally strongly convex cost functional for a coefficient inverse problem, Nonlinear Analysis: Real World Applications, 22, 272-278, 2015 .

[14] F. Berntsson, V.A. Kozlov, L. Mpinganzima and B.O. Turesson, An alternating iterative procedure for the Cauchy problem for the Helmholtz equation, Inverse Problems in Science and Engineering, 22, 45-62, 2014.

[15] L. Bourgeois, A mixed formulation of quasi-reversibility to solve the Cauchy problem for the Laplace's equation, Inverse Problems, 21, 1087-1104, 2005. 
[16] L. Bourgeois, Convergence rates for the quasi-reversibility method to solve the Cauchy problem for Laplace's equation, Inverse Problems, 22, 413-430, 2006.

[17] L. Bourgeois and E. Linéville, The method of quasi-reversibility to solve the Cauchy problems for elliptic partial differential equations, Proceedings Appl. Math. Mech., 7, 1042191-1042102, 2007.

[18] L. Bourgeois, About stability and regularization of ill-posed elliptic Cauchy problems: the case of $C^{1,1}$ domains, ESAIM: M2AN 44, 715-635, 2010.

[19] L. Bourgeois and J. Dardé, About stability and regularization of ill-posed elliptic Cauchy problems: the case of Lipschitz domains, Applicable Analysis, 89, 1745-1768, 2010 .

[20] L. Bourgeois and J. Dardé, A duality-based method of quasi-reversibility to solve the Cauchy problem in the presence of noisy data, Inverse Problems, 26, 095016, 2010.

[21] L. Bourgeois and J. Dardé, A quasi-reversibility approach to solve the inverse obstacle problem, Inverse Problems and Imaging, 4, 351-377, 2010.

[22] L. Bourgeois and J. Dardé, About identification of defects in an elastic-plastic medium from boundary measurements in the antiplane case, Applicable Analysis, 90, 1481-1497, 2011.

[23] L. Bourgeois and J. Dardé, The "exterior approach" to solve the inverse obstacle problem for the Stokes system, Inverse Problems and Imaging, 8, 23-51, 2014.

[24] A.L. Bukhgeim and M.V. Klibanov, Uniqueness in the large of a class of multidimensional inverse problems, Soviet Math. Doklady, 17, 244-247, 1981.

[25] A.L. Bukhgeim, Carleman estimates for Volterra operators and uniqueness of inverse problems, in Non-Classical Problems of Mathematical Physics, pages 54-64, published by Computing Center of the Siberian Branch of the USSR Academy of Science, Novosibirsk, 1981 (in Russian).

[26] A.L. Bukhgeim, Introduction In The Theory of Inverse Problems, VSP, Utrecht, The Netherlands, 2000.

[27] H. Cao, M.V. Klibanov and S.V. Pereverzev, A Carleman estimate and the balancing principle in the quasi-reversibility method for solving the Cauchy problem for the Laplace equation, Inverse Problems, 25, 35005, 2009.

[28] Y.T. Chow and J. Zou, A numerical method for reconstructing the coefficient in a wave equation, Numerical Methods for Partial Differential Equations, published online, DOI: 10.1002/num.21904, 2014.

[29] C. Clason and M.V. Klibanov, The quasi-reversibility method for thermoacoustic tomography in a heterogeneous medim, SIAM J. Sci. Comp., 30, 1-23, 2007.

[30] J. Dardé, A. Hannukainen and N. Hyvönen, An $H_{\text {div }}$-based mixed quasi-reversibility method for solving elliptic Cauchy problems, SIAM J. Numer. Analysis, 51, 21232148, 2013. 
[31] L. Eldén, Numerical solution of the sideways heat equation by difference approximation in time, Inverse Problems, 11, 913-923, 1995.

[32] L. Eldén, F. Berntsson and T. Regińska, Wavelet and Fourier methods for solving the sideways heat equation, SIAM J. Sci. Comp., 21, 2187-2205, 2000.

[33] H. W. Engl, M. Hanke and A. Neubauer, Regularization of Ill-Posed Problems, Kluwer Academic Publishers, Boston, 2000.

[34] D.N. Hào, A noncharacteristic Cauchy problem for linear parabolic equations II: a variational method, Numerical Functional Analysis and Optimization, 13, 541-564, 1992.

[35] D.N. Hào and D. Lesnic, The Cauchy problem for Laplace's equation via the conjugate gradient method, IMA J. Appl. Math., 65, 199-217, 2000.

[36] V. Isakov, Inverse Problems for Partial Differential Equations, Second Edition, Springer, New York, 2006.

[37] V. K. Ivanov, V. V. Vasin and V. P. Tanana, Theory of Linear Ill-Posed Problems and Its Applications, De Gruyter, Berlin, 2002.

[38] S.I. Kabanikhin, Inverse and Ill-Posed Problems, De Gruiter, Leipzig, 2012.

[39] S.I. Kabanikhin and M.A. Shishlenin, Direct and iterative methods of solving of inverse and ill-posed problems, Siberian Electronic Mathematical Reports, 5, 595-608, 2008 .

[40] S.I. Kabanikhin, A.D. Satybaev and M.A. Shishlenin, Direct Methods of Solving Multidimensional Inverse Hyperbolic Problems, VSP, Utrecht, 2004.

[41] S.I. Kabanikhin and M.A. Shishlenin, Numerical algorithm for two-dimensional inverse acoustic problem based on Gel'fand-Levitan-Krein equation, J. Inverse and Ill-Posed Problems, 18, 979-995, 2011.

[42] A.L. Karchevsky, Reformulation of an inverse problem statement that reduces computational costs, Eurasian J. Of Mathematical and Computer Applications, V. 1, issue 2, 4-20, 2013.

[43] M. V. Klibanov, Uniqueness of solutions in the 'large' of some multidimensional inverse problems, in Non-Classical Problems of Mathematical Physics, pages 101-114, 1981, published by Computing Center of the Siberian Branch of the USSR Academy of Science, Novosibirsk (in Russian).

[44] M. V. Klibanov, Inverse problems in the 'large' and Carleman bounds, Differential Equations, 20, 755-760, 1984.

[45] M. V. Klibanov, Inverse problems and Carleman estimates, Inverse Problems, 8, 575-596, 1992.

[46] M. Kazemi and M.V. Klibanov, Stability estimates for ill-posed Cauchy problem involving hyperbolic equation and inequalities, Applicable Analysis, 50, 93-102, 1993. 
[47] M.V. Klibanov and F. Santosa, A computational quasi-reversibility method for Cauchy problems for Laplace's equation, SIAM J. Appl. Math., 51, 1653-1675, 1991.

[48] M.V. Klibanov and J. Malinsky, Newton-Kantorovich method for 3-dimensional potential inverse scattering problem and stability for the hyperbolic Cauchy problem with time dependent data, Inverse Problems, 7, 577-596, 1991.

[49] M.V. Klibanov and Rakesh, Numerical solution of a timelike Cauchy problem for the wave equation, Math. Meth. in Appl. Sci., 15, 559-570, 1992.

[50] M.V. Klibanov, Global convexity in a three-dimensional inverse acoustic problem, SIAM J. Math. Analysis, 28, 1371-1388, 1997.

[51] M.V. Klibanov, Global convexity in diffusion tomography, Nonlinear World, 4, 247265, 1997.

[52] M.V. Klibanov and A. Timonov, Carleman Estimates for Coefficient Inverse Problems and Numerical Applications, VSP, Utrecht, The Netherlands, 2004.

[53] M.V. Klibanov, Estimates of initial conditions of parabolic equations and inequalities via lateral Cauchy data, Inverse Problems, 22, 495-514, 2006.

[54] M.V. Klibanov, A.V. Kuzhuget, S.I. Kabanikhin and D.V. Nechaev, A new version of the quasi-reversibility method for the thermoacoustic tomography and a coefficient inverse problem, Applicable Analysis, 87, 1227-1254, 2008.

[55] M.V. Klibanov, Thermoacoustic tomography with an arbitrary elliptic operator, Inverse Problems, 29, 025014, 2013.

[56] M.V. Klibanov, Carleman estimates for global uniqueness, stability and numerical methods for coefficient inverse problems, J. Inverse and Ill-Posed Problems, 21, 477$560,2013$.

[57] M.V. Klibanov and N.T. Thành, Recovering of dielectric constants of explosives via a globally strictly convex cost functional, arxiv: 1408.0583, 2014.

[58] V.A. Kozlov, V.G. Maz'ya and A.V. Fomin, An iterative method for solving the Cauchy problem for elliptic equations, U.S.S.R. Comput. Math. and Math. Phys. 31, 45-52, 1991.

[59] A.V. Kuzhuget, L. Beilina, M.V. Klibanov, A. Sullivan, L. Nguyen and M.A. Fiddy, Blind experimental data collected in the field and an approximately globally convergent inverse algorithm, Inverse Problems, 28, 095007, 2012.

[60] O.A. Ladyzhenskaya, Boundary Value Problems of Mathematical Physics, Springer, New York, 1985.

[61] I. Lasiecka, R. Triggiani and X. Zhang, Global uniqueness, observability and stabilization of non-conservative Schrödinger equations via pointwise Carleman estimates. Part I: $H^{1}(\Omega)$-estimates, J. of Inverse and Ill-Posed Problems, 12, 1-81, 2004. 
[62] I. Lasiecka, R. Triggiani and X. Zhang, Global uniqueness, observability and stabilization of non-conservative Schrödinger equations via pointwise Carleman estimates. Part II: $L_{2}(\Omega)$-estimates, J. of Inverse and Ill-Posed Problems, 12, 182-231, 2004.

[63] R. Lattes and J.-L. Lions, The Method of Quasireversibility: Applications to Partial Differential Equations, Elsevier, New York, 1969.

[64] M.M. Lavrentiev, V.G. Romanov and S.P. Shishatskii, Ill-Posed Problems of Mathematical Physics and Analysis, AMS, Providence, R.I., 1986.

[65] J. Li, J. Xie and J. Zou, An adaptive finite element reconstruction of distributed fluxes, Inverse Problems, 27, 075009, 2011.

[66] Y. Liu, J. Su, Z.-J. Lin, S. Teng, A. Rhoden, N. Pantong, and H. Liu, Reconstructions for continuous-wave diffuse optical tomography by a globally convergent method, $J$. of Applied Mathematics and Physics, 2, 204-213, 2014.

[67] E. Ozbilge, Convergence theorem for a numerical method of a 1D coefficient inverse problem, Applicable Analysis, 93, 1614-1625, 2014.

[68] L.E. Payne, Improperly Posed Problems in Partial Differential Equations, SIAM, Philadelphia, PA, 1975.

[69] K.-D. Phung, Remarques sur l'observabilité pour l'équation de Laplace, EASIM Proceedings "Control Optimization and Calculus Variations", 9, 133-143, 2003.

[70] N.T. Thành, L. Beilina, M. V. Klibanov and M. A. Fiddy, Reconstruction of the refractive index from experimental backscattering data using a globally convergent inverse method, SIAM J. Sci. Comp., 36, B273-B293, 2014.

[71] A.N. Tikhonov, A.V. Goncharsky, V.V. Stepanov and A.G. Yagola, Numerical Methods for the Solution of Ill-Posed Problems, Kluwer, London, 1995.

[72] R. Triggiani and P.F. Yao, Carleman estimates with no lower order terms for general Riemannian wave equations. Global uniqueness and observability in one shot, Appl. Math. and Optimization, 46, 331-375, 2002.

[73] A.G. Yagola, A.S. Leonov and V.N. Titarenko, Data errors and an error estimation for ill-posed problems, Inverse Problems in Science and Engineering, 10, 117-129, 2002.

[74] M. Yamamoto, Carleman estimates for parabolic equations. Topical Review. Inverse Problems, 25, 123013, 2009. 